\title{
Self-Effect in Expanding Electron Beam Plasma
}

\author{
Manuel Garcia
}

May 7, 1999

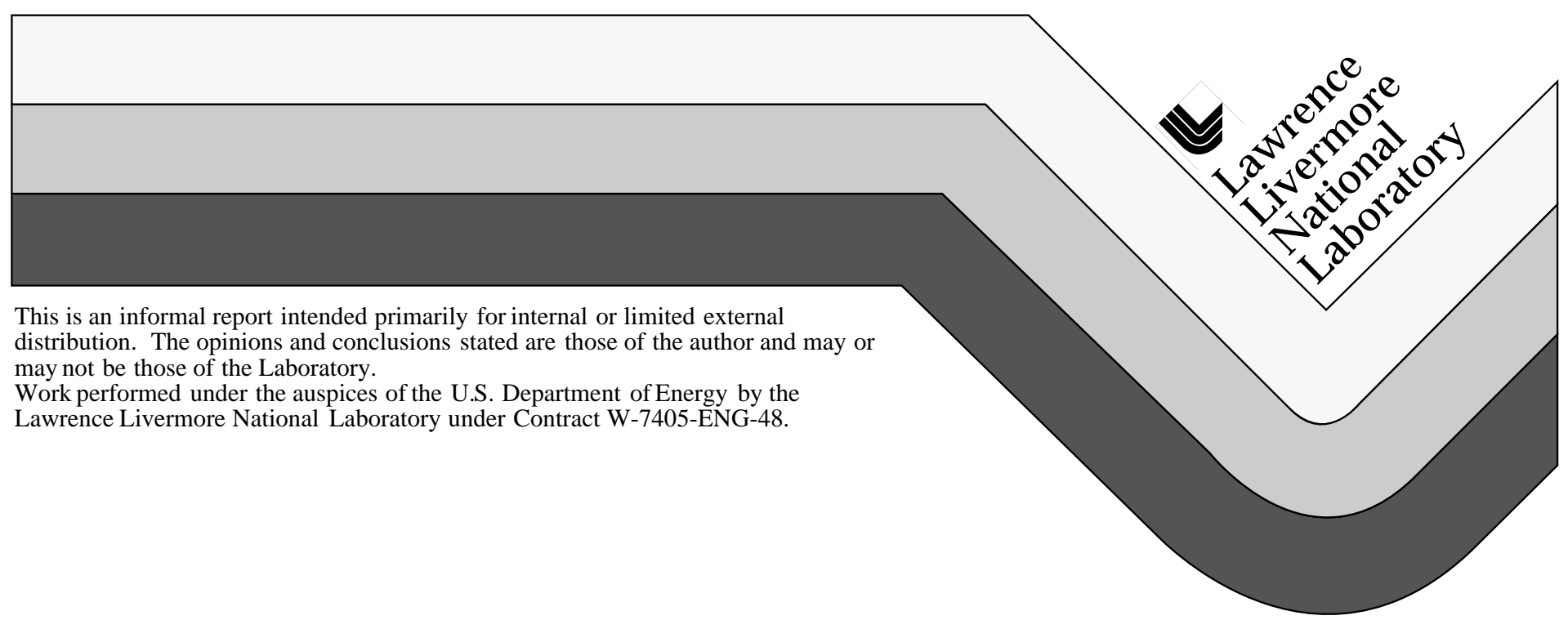




\section{DISCLAIMER}

This document was prepared as an account of work sponsored by an agency of the United States Government. Neither the United States Government nor the University of California nor any of their employees, makes any warranty, express or implied, or assumes any legal liability or responsibility for the accuracy, completeness, or usefulness of any information, apparatus, product, or process disclosed, or represents that its use would not infringe privately owned rights. Reference herein to any specific commercial product, process, or service by trade name, trademark, manufacturer, or otherwise, does not necessarily constitute or imply its endorsement, recommendation, or favoring by the United States Government or the University of California. The views and opinions of authors expressed herein do not necessarily state or reflect those of the United States Government or the University of California, and shall not be used for advertising or product endorsement purposes.

This report has been reproduced directly from the best available copy.

Available to DOE and DOE contractors from the Office of Scientific and Technical Information P.O. Box 62, Oak Ridge, TN 37831

Prices available from (423) 576-8401

Available to the public from the National Technical Information Service

U.S. Department of Commerce 5285 Port Royal Rd., Springfield, VA 22161 


\title{
Self-effect in expanding electron beam plasma
}

\author{
Manuel Garcia \\ 7 May 1999
}

Lawrence Livermore National Laboratory, L-153, POB 808 Livermore, CA 94551-0808 USA

garcia22@llnl.gov, (925) 422-6017, FAX (925) 423-5080

An analytical model of plasma flow from a metal plate hit by an intense, pulsed, electron beam aims to bridge the gap between radiation-hydrodynamics simulations and experiments, and to quantify the self-effect of the electron beam penetrating the flow. Does the flow disrupt the tight focus of the initial electron bunch, or later pulses in a train? This work aims to model the spatial distribution of plasma speed, density, degree of ionization, and magnetization to inquire. The initial solid density, several eV plasma expands to $1 \mathrm{~cm}$ and $10^{-4}$ relative density by $2 \mu \mathrm{s}$, beyond which numerical simulations are imprecise. Yet, a Faraday cup detector at the ETA-II facility is at $25 \mathrm{~cm}$ from the target and observes the flow after $50 \mu \mathrm{s}$. The model helps bridge this gap.

The expansion of the target plasma into vacuum is so rapid that the ionized portion of the flow departs from local thermodynamic equilibrium. When the temperature (in $\mathrm{eV}$ ) in a parcel of fluid drops below $V_{i} \times[(2 \gamma-2) /(5 \gamma+17)]$, where $V_{i}$ is the ionization potential of the target metal $(7.8 \mathrm{eV}$ for tantalum), and $\gamma$ is the ratio of specific heats ( $5 / 3$ for atoms), then the fractional ionization and electron temperature in that parcel remain fixed during subsequent expansion. The freezing temperature as defined here is $\mathrm{V}_{\mathrm{i}} / 19$.

The balance between the self-pinching force and the space charge repulsion of an electron beam changes on penetrating a flow:

(i) the target plasma cancels the space-charge field,

(ii) internal eddy currents arise to counter the magnetization of relativistic electrons, and

(iii) electron beam heating alters the flow magnetization by changing the plasma density gradient and the magnitude of the conductivity. These cause beam expansion (besides scattering). 
The evolution of the total magnetic induction $\mathbf{B}$ (beam-intarget) is given by

$$
\begin{aligned}
\frac{\partial \mathbf{B}}{\partial t}= & {\left[\frac{\nabla^{2} \mathbf{B}}{\sigma \mu}+\operatorname{curl}(\mathbf{v} \times \mathbf{B})+\frac{\nabla \sigma}{\sigma} \times \frac{\operatorname{curl}(\mathbf{B})}{\sigma \mu}\right]+} \\
& {\left[\frac{\nabla k T_{\mathrm{e}} \times \nabla n_{\mathrm{e}}}{\mathrm{en} n_{\mathrm{e}}}-\frac{\nabla^{2} \mathbf{B}_{0}}{\sigma \mu}-\frac{\nabla \sigma}{\sigma} \times \frac{\operatorname{curl}\left(\mathbf{B}_{0}\right)}{\sigma u}\right], }
\end{aligned}
$$

where $\mathbf{B}_{0}$ is the beam magnetic induction. The generated magnetic field has the opposite polarity to the self-field of the electron beam. Large local values arise where the density gradients are large: at the leading edge of the cloud, and at the surface of the plate. The second square bracket includes the thermal source term powered by beam heating, and the internal eddy current terms powered by the beam magnetization. Heating raises $\sigma$ when a beam enters a cloud, which slows the diffusive loss of B and diminishes the first Bdot bracket. The second B-dot bracket appears because significant $\nabla T_{\mathrm{e}}$ and $\mathrm{B}_{0}$ arise. When a beam pulse ends, expansion cooling dissipates $\nabla T_{\mathrm{e}}$, and both $\mathrm{B}_{0}$ and the second Bdot bracket disappear. The diminution of $\sigma$ raises the first Bdot bracket and gives rise to a large $\partial \mathrm{B} / \partial t$, possibly observable as a "surface flashover" across the front of the cloud.

\section{Electron beam expansion by target heating}

This report describes how an electron beam expands as a result of heating the target foil it is penetrating. This report completes work that evolved through several stages, which are described in [1], [2], [3], [4], [5] and [6]. The significant finding of this work is that the appropriate form of Faraday's law is

$$
\begin{aligned}
\frac{\partial \mathbf{B}}{\partial t}= & {\left[\frac{\nabla^{2} \mathbf{B}}{\sigma u}+\operatorname{curl}(\mathbf{v} \times \mathbf{B})+\frac{\nabla \sigma}{\sigma} \times \frac{\operatorname{curl}(\mathbf{B})}{\sigma u}\right]+} \\
& {\left[\frac{\nabla \mathrm{k} T_{\mathrm{e}} \times \nabla n_{\mathrm{e}}}{\mathrm{en} n_{\mathrm{e}}}-\frac{\nabla^{2} \mathbf{B}_{0}}{\sigma u}-\frac{\nabla \sigma}{\sigma} \times \frac{\operatorname{curl}\left(\mathbf{B}_{0}\right)}{\sigma u}\right], }
\end{aligned}
$$


where $\mathbf{B}$ is the magnetic induction generated in a flow of conductivity $\sigma$, of electron temperature and electron density gradients $\nabla T_{\mathrm{e}}$ and $\nabla n_{\mathrm{e}}$, and of velocity $\mathbf{v}$, and where $\mathbf{j}_{0}=\operatorname{curl}\left(\mathbf{B}_{0} / \mu\right)$ is the current density of the relativistic electron beam. In equation (1), $\mathrm{k}$ is Boltzmann's constant, e is the elementary charge, $\mu$ is the magnetic permeability, and $t$ is the time parameter. As an electron beam heats a target, $n_{\mathrm{e}}, T_{\mathrm{e}}, \sigma, \mathrm{v}, \nabla T_{\mathrm{e}}$, and $\nabla n_{\mathrm{e}}$ all change and the target can magnetize quickly. When the beam is absent and electron density and temperature gradients align, equation (1) reduces to the usual Faraday's law of MHD. The following sections will describe the thermal expansion of the target, the nonequilibrium nature of this ionized flow, and the MHD consequences of an electron beam heat source within this flow.

\section{Thermal expansion of the target - the source}

By assumption, the electron beam instantly heats a cylindrical volume $V_{0}$ through the target to a uniform temperature $T_{0}(0)$. Matter flows out of volume $V_{0}$ through the total disc area $A_{0}=2 \pi r_{0}^{2}$ at a local sonic velocity $c *(t)=[\gamma \mathrm{k} T *(t) / \mathrm{m}]^{1 / 2}$, for atom mass $\mathrm{m}$. As the discharge progresses, the density $\rho_{0}(t)$ and temperature $T_{0}(t)$ in the source volume $V_{0}$ decrease. The rate of change of the source volume density $\rho_{0}(t)$ is

$$
\frac{d \rho_{0}}{d t}=-\frac{m^{\prime}}{V_{0}}=-\frac{\rho_{*} C_{*} A_{0}}{V_{0}}=-\left(\frac{\rho_{0}}{\left(1+\frac{\gamma-1}{2}\right)^{\frac{1}{\gamma-1}}}\right)\left(\frac{c_{0}}{\sqrt{1+\frac{\gamma-1}{2}}}\right) \frac{A_{0}}{V_{0}},
$$

where $m^{\prime}$ is the mass flow rate, which is given as the mass flux through the cross-sections where the flow is just sonic. The mass flux at the sonic sections is related to conditions in the source volume by assuming the flow to be that of an ideal perfect gas with a ratio of specific heats $\gamma$. The speed of sound in the source volume is $c_{0}(t)=\left[\gamma \mathrm{k} T_{0}(t) / \mathrm{m}\right]^{1 / 2}$. See [7] and [8] for descriptions of compressible flow.

This model assumes that the source volume equilibrates quickly to changing conditions, and it is always uniform. The initial temperature is

$$
T_{0}(0)=\left(\frac{\gamma-1}{\gamma}\right)\left(\frac{\mathrm{m}}{\mathrm{k}}\right)\left(\frac{\Delta U}{\rho_{0}(0) V_{0}}-\Delta H_{V}\right)+T_{\mathrm{v}}
$$


where $\Delta U$ is the heat energy deposited by the electron beam, $\Delta H_{\mathrm{v}}$ is the heat of vaporization from solid density, and $T_{\mathrm{V}}$ is the temperature at vaporization. The temperature and density at time $t$ in the source volume are related to initial conditions $T_{0}(0)$ and $\rho_{0}(0)$ by

$$
\frac{T_{0}(t)}{\rho_{0}(t)^{\gamma-1}}=\frac{T_{0}(0)}{\rho_{0}(0)^{\gamma-1}} \text {. }
$$

Applying (4) within $c_{0}(t)$ in equation (2) leads to

$$
\frac{d \rho_{0}}{d t}=-\left(\frac{2}{\gamma+1}\right)^{\frac{1}{2}\left(\frac{\gamma+1}{\gamma-1}\right)} \frac{\rho_{0} c_{0} A_{0}}{V_{0}}=-\left(\frac{2}{\gamma+1}\right)^{\frac{1}{2}\left(\frac{\gamma+1}{\gamma-1}\right)} \frac{\rho_{0}(0) c_{0}(0) A_{0}}{V_{0}}\left(\frac{\rho_{0}(t)}{\rho_{0}(0)}\right)^{\left(\frac{\gamma+1}{2}\right)},
$$

and this integrates to

$$
\begin{aligned}
& \frac{\rho_{0}(t)}{\rho_{0}(0)}=\left(\frac{1}{1+\frac{t}{t_{0}}}\right)^{\frac{2}{\gamma-1}} \\
& t_{0}=\frac{V_{0}}{c_{0}(0) A_{0}}\left(\frac{2}{\gamma-1}\right) \sqrt{\left(\frac{\gamma+1}{2}\right)^{\frac{\gamma+1}{\gamma-1}}} .
\end{aligned}
$$

The equation for the temperature follows from (4)

$$
\frac{T_{0}(t)}{T_{0}(0)}=\left(\frac{1}{1+\frac{t}{t_{0}}}\right)^{2}
$$

Figure 1 shows the decay of source number density for a tantalum volume $100 \mu \mathrm{m}$ long and $0.8 \mathrm{~mm}$ in diameter, which was heated to $9 \mathrm{eV}$ by a heating impulse of 10 joules. Figure 2 shows the corresponding decay of source temperature. This source volume is essentially a void by $10 \mu \mathrm{s}$. 


\section{Thermal expansion of the target - the external flow}

Flow from the source into the external vacuum is assumed to be one dimensional. The shape of a flow front, as it advances from a sonic disc $A_{0} / 2$, is assumed to form according to Huygens' principle with the local supersonic speed. Figure 3 is a schematic showing these flow fronts. The area of such a flow front that crosses the normal to $A_{0} / 2$ at distance $z$ is

$$
A(z)=2 \pi\left(z^{2}+\frac{\pi}{2} r_{0} z\right)+\pi r_{0}^{2} .
$$

$A(z)$ evolves from a disc to a hemisphere with increasing $z$.

The Mach number of one dimensional flow is related to the area ratio by

$$
M^{2}=\left(\frac{A_{0}}{2 A(z)}\right)^{2}\left(\frac{2}{\gamma+1}\right)_{\gamma-1}^{\frac{\gamma+1}{\gamma+1}}\left(1+\frac{\gamma-1}{2} M^{2}\right)^{\frac{\gamma+1}{\gamma-1}} \text {. }
$$

The ratios of flow properties at front $A(z)$ to the respective properties in the source are parametrized by Mach number $M(z)$ and called streamtube-area relations, see [7] and [8]. The flight time to coordinate $z$ of a parcel of fluid leaving the source at time $t$ is

$$
\tau(z, t)=\int_{0}^{Z} \frac{\sqrt{1+\frac{\gamma-1}{2} M(\xi)^{2}}}{c_{0}(t) M(\zeta)} d \xi
$$

The temperature, number density, and velocity of the flow at coordinate $z$ as functions of time are:

$$
\begin{aligned}
& T(z, t+\tau)=\frac{T_{0}(t)}{1+\frac{\gamma-1}{2} M(z)^{2}} \\
& N(z, t+\tau)=\frac{N_{0}(t)}{\left(1+\frac{\gamma-1}{2} M(z)^{2}\right)^{\frac{1}{\gamma-1}}}
\end{aligned}
$$




$$
v(z, t+\tau)=\frac{c_{0}(t) M(z)}{\sqrt{1+\frac{\gamma-1}{2} M(z)^{2}}} .
$$

Figure 4 shows the number density profile along $z$ at $t+\tau(z, t)=275 \mathrm{~ns}$ for the tantalum flow of Figures 1 and 2. Figure 5 shows the corresponding temperature profile for the tantalum fluid, and Figure 6 shows the velocity profile.

\section{The transition to nonequilibrium ionization}

Flow expands so rapidly into the external vacuum that the ionized portion of the fluid departs from local thermodynamic equilibrium. This cold supersonic exhaust will have a much higher degree of ionization, and of higher electron temperature, than would be expected from a gas in thermodynamic equilibrium at the local temperature of the neutral flow. The phenomenon of sudden expansion of a gas cloud into vacuum is described in several sections of the two-volume work by Zel'dovich and Raizer, [9]. The criterion I use for estimating the point in the flow where plasma ceases to be in equilibrium is in principle that proposed by Bray, [10] and [11], and discussed extensively by Vincenti and Kruger [12]. My earlier analysis of frozen flow, in [2], assumed a steady state or slowly varying source. The analysis here includes the effect of source decay.

From the perspective of a frame of reference moving with the massaverage velocity of the flow, the time rate of change of plasma density within a small parcel of fluid is

$$
\frac{\partial n_{\mathrm{e}}}{\partial t}=\alpha n_{\mathrm{e}}-P n_{\mathrm{e}}^{2}
$$

for ionization rate $\alpha n_{\mathrm{e}}$ and recombination rate $P n_{\mathrm{e}} n_{+}$. Here, $\alpha$ is the ionization rate coefficient, $P$ is the recombination rate coefficient, $n_{\mathrm{e}}$ is the electron number density, and $n_{+}$is the ion number density. Only single ionization is considered because the multiply-ionized source plasma quickly recombines to a mixture of singly-ionized plasma and metal vapor as the flow begins. Calculations by DeVolder, [13] and [14], show that the Saha model of thermal ionization adequately describes this early, equilibrium state of the plasma flow. A small digression on the Saha equation follows. 
The equation developed by Saha (1920) for the equilibrium concentration of plasma in a gas at temperature $T$ in ${ }^{\circ} \mathrm{K}$ is

$$
\frac{\left(\frac{n_{+}}{N}\right)^{2}}{1-\left(\frac{n_{+}}{N}\right)^{2}} p=\frac{2 \mathrm{~g}_{+}}{\mathrm{g}_{\mathrm{N}}}\left(\frac{2 \pi \mathrm{m}_{\mathrm{e}}}{\mathrm{h}^{2}}\right)^{3 / 2}(\mathrm{k} T)^{5 / 2} \mathrm{e}^{-\mathrm{eV} \mathrm{i} / \mathrm{k} T}
$$

Equation (15) is in the form shown by Cobine [15] and also by Vincenti and Kruger [12]. Here electron and ion densities are assumed equal $\left(n_{\mathrm{e}}=n_{+}\right)$, the gas pressure is $p=\mathrm{kNT}$ with number density $N=\rho / \mathrm{m}_{N}$ (for atomic mass $m_{N}$ ), the factor involving the ion degeneracy $g_{+}$and neutral degeneracy $g_{N}$ is assumed equal to one, $V_{i}$ is the ionization potential in $\mathrm{eV}$, $\mathrm{k}$ is Boltzmann's constant, $\mathrm{h}$ is Planck's constant, $\mathrm{m}_{\mathrm{e}}$ is the electron mass, and e is the elementary charge. For $T$ in units of eV the equilibrium concentration is

$$
\begin{aligned}
& \frac{\left(\frac{n_{+}}{N}\right)^{2}}{1-\left(\frac{n_{+}}{N}\right)^{2}}=\frac{1}{N} \frac{2 \mathrm{~g}_{+}}{\mathrm{g}_{\mathrm{N}}}\left(\frac{2 \pi \mathrm{m}_{\mathrm{e}} \mathrm{e}}{\mathrm{h}^{2}}\right)^{3 / 2} T^{3 / 2} \mathrm{e}^{-\mathrm{V}_{\mathrm{i}} / T} \equiv K(T, N) \\
& \frac{n_{+}}{N}=\sqrt{\frac{K(T, N)}{1+K(T, N)}} \equiv \phi(T, N) .
\end{aligned}
$$

This restatement of Saha's equation is consistent with forms shown by Tanenbaum [16], and Mitchner and Kruger [17]. For $T$ in $\mathrm{eV}$ and $N$ in $\mathrm{cm}^{-3}$, the function $K(T, N)$ is

$$
K(T, N)=\left(3.0183 \times 10^{21} \frac{1}{\mathrm{~cm}^{3} \mathrm{eV}^{3 / 2}}\right) \frac{1}{N} T^{3 / 2} \mathrm{e}^{-\mathrm{V}_{\mathrm{i}} / T} .
$$

Now we return to the discussion of nonequilibrium flow.

A parcel in local thermodynamic equilibrium will have a concentration of plasma $n_{\mathrm{e}}=n_{+}=\alpha / P$, where the rate coefficients $\alpha$ and $P$ each depend on $T$ and $N$, see equation (14). If the plasma density is perturbed from equilibrium by a small quantity $\eta$, then 


$$
\begin{aligned}
& n_{\mathrm{e}}=\frac{\alpha}{P}+\eta \\
& \frac{\partial n_{\mathrm{e}}}{\partial t}=\alpha n_{\mathrm{e}}-P n_{\mathrm{e}}^{2} \rightarrow \frac{\partial \eta}{\partial t}=-\alpha \eta-P \eta^{2} \\
& \eta(t) \approx \eta(0) \mathrm{e}^{-\alpha t},
\end{aligned}
$$

where small terms of second order are neglected. The ionization rate coefficient is the measure of the responsiveness of the fluid to perturbations from equilibrium of its plasma density.

The equation of continuity for the plasma species from the perspective of a static frame of reference is

$$
\frac{\partial n_{\mathrm{e}}}{\partial t}+\nabla \cdot n_{\mathrm{e}} \mathbf{v}=0
$$

where $\mathbf{v}$ is the mass-average velocity, plasma diffusion is neglected, and there are no external sources or sinks of plasma. In equilibrium flow the local rate-dependent processes compensate for any local inflow or outflow of plasma density, so equation (19) can be restated as

$$
\alpha n_{\mathrm{e}}-P n_{\mathrm{e}}^{2}+\nabla \cdot n_{\mathrm{e}} \mathbf{v}=0 .
$$

Rate-dependent processes are so fast in equilibrium flow that they easily adjust to any changes introduced by transport. If $L_{\mathrm{n}}$ is a length scale for the variation of density, and $V$ is a characteristic flow velocity, then $\alpha L_{\mathrm{n}} / V>>1$. This nondimensional parameter is the exponential in equation (18) when $t=L_{\mathrm{n}} / V$, the time scale for the variation of density.

The temperature and density drop so precipitously during the rapid expansion into the vacuum, that both the ionization and recombination rate coefficients decrease by orders of magnitude over a short distance. The flow far downstream from the source is a simple geometric expansion

$$
\sqrt{\frac{2}{\gamma-1}} \frac{c_{0}\left(t-\Delta t_{\mathrm{R}}\right)}{R^{2}} \frac{\partial}{\partial R}\left(R^{2} n_{\mathrm{e}}\right)=0,
$$


where $\Delta t_{\mathrm{R}}$ is the flight time to radial coordinate $R$. Here, $\alpha L_{\mathrm{n}} / V \ll 1$ and the plasma density and electron temperature are "frozen." The frozen plasma density is well above that expected from local thermodynamic conditions because the three-body recombination reaction no longer occurs in the cold and rarefied flow.

The transition from equilibrium flow to frozen flow is sudden, and it can be approximated as occurring at a single hemispherical cross section of the expansion. At this transition point, recombination is negligible and the rate of change due to ionization is comparable to the rate of change due to transport, or equivalently $\alpha L_{n} / V=1$. The criterion for determining the point of transition is

$$
\alpha n_{\mathrm{e}}+\nabla \cdot n_{\mathrm{e}} \mathbf{V}=0=\alpha n_{\mathrm{e}}+\frac{1}{R^{2}} \frac{\partial}{\partial R}\left(R^{2} n_{\mathrm{e}} V\right),
$$

an idea originally proposed by Bray, see references [10], [11], and [12]. Note that the quantities in equation (22) are calculated on the basis of equilibrium flow. Conditions prior to the transition are taken to be in complete equilibrium, and conditions after the transition are assumed frozen.

\section{Frozen plasma flow}

An explicit formula for the coordinate $Z_{\mathrm{B}}$ at the point of transition is derived from equation (22) by using the stream-tube-area relations, equations (9), (10), (11), (12), and (13) to specify $T(z, t+\tau), N(z, t+\tau)$, and $v(z, t+\tau)$, as well as the Saha equation for $n_{\mathrm{e}}$, and a formula for the ionization coefficient $\alpha(T, N)$. As a matter of convenience in deriving a formula for $z_{\mathrm{B}}$, coordinate $z$ will be taken as the radius of purely hemispherical flow fronts. This means that $\left(\mathrm{A}_{0} / 2\right) / A(Z)$ in equation $(9)$ is replaced by $\left(\pi r_{0}^{2}\right) /\left(2 \pi z^{2}\right)$, which is inaccurate for $z<r_{0}$. If greater accuracy is desired then the analysis below can be repeated with the $A(z)$ of equation (8) in the Mach number-area relation.

The transition from equilibrium to frozen flow occurs when

$$
\frac{\partial}{\partial Z}\left(v n_{\mathrm{e}} Z^{2}\right)+\alpha n_{\mathrm{e}} Z^{2}=\frac{\partial}{\partial Z}\left(v Z^{2} N \phi\right)+\alpha Z^{2} N \phi=0 .
$$


At the point of transition $Z=Z_{\mathrm{B}}$, and (23) becomes

$$
\begin{aligned}
& \frac{-2}{Z_{\mathrm{B}}}=\frac{\alpha}{V}+\frac{1}{\phi} \frac{\partial \phi}{\partial Z}+\frac{1}{V} \frac{\partial V}{\partial Z}+\frac{1}{N} \frac{\partial N}{\partial Z} \\
& \frac{-2}{Z_{\mathrm{B}}}=\frac{\alpha}{V}+\frac{1}{\phi}\left(\frac{\partial \phi}{\partial T} \frac{\partial T}{\partial Z}+\frac{\partial \phi}{\partial N} \frac{\partial N}{\partial Z}\right)+\frac{1}{V} \frac{\partial V}{\partial Z}+\frac{1}{N} \frac{\partial N}{\partial Z} .
\end{aligned}
$$

Notice from equations (9) through (13) that the spatial derivatives of $T, N$, and $v$ also involve time. It is convenient to reference the time of departure of a parcel of fluid from the source, $t$, to its time of arrival at coordinate $z$.

Flow that leaves the source at time $t$ arrives at coordinate $z$ at time

$$
\begin{aligned}
& t_{\mathrm{z}}=t+\tau(z, t)=t+\frac{1}{c_{0}(t)} \int_{0}^{z} \frac{\sqrt{1+\frac{\gamma-1}{2} M(\zeta)^{2}}}{M(\zeta)} d \zeta=t+\frac{\left(1+\frac{t}{t_{0}}\right)}{c_{0}(0)} l_{\mathrm{z}}(z) \\
& t_{\mathrm{z}}=t\left(1+\frac{t_{\mathrm{z} 0}}{t_{0}}\right)+t_{\mathrm{z} 0}=t\left(1+\frac{l_{\mathrm{z}}(z)}{c_{0}(0) t_{0}}\right)+\frac{l_{\mathrm{z}}(z)}{c_{0}(0) t_{0}}
\end{aligned}
$$

Now, make $t_{\mathrm{z}}$ and $z$ the independent variables, and find the corresponding time at the source as

$$
t\left(t_{\mathrm{z}}, z\right)=\frac{t_{\mathrm{z}}-t_{\mathrm{z} 0}}{\left(1+\frac{t_{\mathrm{z} 0}}{t_{0}}\right)} .
$$

The stream-tube-area relations for $T, N$, and $v$ are now given by equations (11), (12), and (13) with $t_{\mathrm{z}}$ in place of $t+\tau$ on the left, and equation (26) for $t$ on the right.

The derivatives of $T, N$, and $V$ with respect to $Z$, required in equation (24), will include terms proportional to $\partial t / \partial z$ that arise from the differentiation of $T_{0}(t), N_{0}(t)$, and $c_{0}(t)$. In addition, terms proportional to $d M / d z$ will also arise. Consider the derivative $\partial T\left(z, t_{\mathrm{z}}\right) / \partial z$ as an example, 


$$
\frac{\partial T}{\partial Z}=\frac{\left(1+\frac{\gamma-1}{2} M^{2}\right) \frac{d T_{0}}{d t} \frac{\partial t}{\partial Z}-T_{0}(t)\left([\gamma-1] M \frac{d M}{d z}\right)}{\left(1+\frac{\gamma-1}{2} M^{2}\right)^{2}} .
$$

Equation (27) depends on the source temperature history and its rate of change, on the Mach number and Mach number gradient, and on $\partial t / \partial z$. Similar equations result for $\partial N\left(z, t_{\mathrm{z}}\right) / \partial Z$ and $\partial v\left(z, t_{\mathrm{z}}\right) / \partial z$. The gradients of $t$ and $M$ are:

$$
\begin{aligned}
& \frac{\partial t}{\partial z}=\frac{\left(1+\frac{t_{z}}{t_{0}}\right)\left(\sqrt{\frac{\gamma+1}{2}}-\frac{1}{M(z)} \sqrt{1+\frac{\gamma-1}{2} M(z)^{2}}\right)}{c_{0}(0)\left(1+\frac{t_{z 0}(z)}{t_{0}}\right)^{2}}, \\
& \frac{d M}{d z}=\frac{1+\frac{\gamma-1}{2} M(z)^{2}}{(\gamma+1) Z M(z)\left[1-\left(\frac{z}{r_{0}}\right)^{4}\left(\frac{\gamma+1}{2+(\gamma-1) M(z)^{2}}\right)^{\frac{2}{\gamma-1}}\right]} .
\end{aligned}
$$

The last two derivatives needed in equation (24) for the point of transition are the partials of the ionization fraction $\phi$ with respect to temperature and density:

$$
\begin{aligned}
& \frac{\partial \phi}{\partial T}=\frac{\left(1-\phi^{2}\right)}{2} \frac{\phi}{T}\left(\frac{3}{2}+\frac{\mathrm{V}_{\mathrm{i}}}{T}\right), \\
& \frac{\partial \phi}{\partial N}=-\frac{\left(1-\phi^{2}\right)}{2} \frac{\phi}{N} .
\end{aligned}
$$

By evaluating equation (24) using the results shown up to now, the point of transition $z_{\mathrm{B}}$ is found as the root for $z$ in 


$$
\begin{aligned}
& Z=\frac{2 v(z, \tau(z, 0))}{\alpha}\left\{\frac{2\left(\gamma+\frac{1}{M^{2}}\right)+\left(1-\phi^{2}\right)\left[(\gamma-1)\left(\frac{3}{2}+\frac{V_{\mathrm{i}}}{T}\right)-1\right]}{(\gamma+1)\left[4-\frac{\gamma+1}{M^{2}\left(2+(\gamma-1) M^{2}\right)}\right]}-1\right\} \frac{1}{S(z, t)}, \\
& S(z, t)=\left[1+\frac{t}{t_{0}}-\frac{\left[1+\frac{1+\phi^{2}}{\gamma-1}+\left(1-\phi^{2}\right)\left(\frac{3}{2}+\frac{V_{\mathrm{i}}}{T}\right)\right]}{\alpha t_{0}\left(1+\frac{\tau(z, 0)}{t_{0}}\right)}\left[\sqrt{\frac{(\gamma+1) M^{2}}{2+(\gamma-1) M^{2}}}-1\right]\right] .
\end{aligned}
$$

If the source is steady (infinitely long) then $t_{0}$ is infinite and $S(z, t)=1$. In the limit of a steady source, $M \rightarrow \infty$, and $\phi \rightarrow 0$, the point of transition occurs at

$$
Z=\frac{2 V(z, \tau(z, 0))}{\alpha}\left\{\frac{\gamma+(\gamma-1)\left(\frac{5}{2}+\frac{\mathrm{V}_{\mathrm{i}}}{T}\right)}{4(\gamma+1)}-1\right\} .
$$

As transition occurs where $\alpha z / v(z, \tau(z, 0))=1$, the temperature at the freezing point in the limit described by (32) is given by

$$
\frac{\mathrm{V}_{\mathrm{i}}}{T}=\frac{5 \gamma+17}{2(\gamma-1)}
$$

which equals 19 for $\gamma=5 / 3$. Thus, a steady tantalum flow with an ionization potential of $\mathrm{V}_{\mathrm{i}}=7.8 \mathrm{eV}$ will experience ionization freezing as the temperature drops below $0.41 \mathrm{eV}$.

Unsteady flow will freeze at a higher temperature. Consider $S(z, t)$ in the high Mach number, low ionization fraction limit,

$$
S(z, t)=\left[1+\frac{t}{t_{0}}-\frac{\frac{1}{\gamma-1}+\frac{5}{2}+\frac{V_{\mathrm{i}}}{T}}{\alpha t_{0}+1}\left[\sqrt{\frac{\gamma+1}{\gamma-1}}-1\right]\right] .
$$

For $S(z, t)$ to be positive we must have $\left(\mathrm{V}_{\mathrm{i}} / T\right) \approx \alpha t_{0}>3$. Basically, if the source is decaying at a rate comparable to the ionization rate then the flow 
is inherently nonequilibrium and there is no transition in positive space from thermal equilibrium to freezing. The temperature at the freezing point for positive $S(z, t)$ in this limiting case is

$$
\frac{\mathrm{V}_{\mathrm{i}}}{T}=\frac{4(\gamma+1) S+\gamma+13}{2(\gamma-1)}
$$

which equals 11 for $S=0$ and $\gamma=5 / 3$. With a hot source at early time $\alpha t_{0}>\left(\mathrm{V}_{\mathrm{i}} / T\right)$ and $S \approx 1$. As time progresses, $\alpha t_{0}$ drops and $S$ approaches 0 . Thus, an unsteady tantalum flow in this limiting case could have a freezing point that increased in temperature from $0.41 \mathrm{eV}$ to $0.71 \mathrm{eV}$ while approaching the source from positive space (more precisely, $z_{\mathrm{B}} \rightarrow 0$ with increasing characteristic lines of the flow).

A parcel of fluid that crosses $Z_{\mathrm{B}}\left(t+\tau\left(Z_{\mathrm{B}}, t\right)\right)$ is assumed to have its electron temperature fixed to $T_{\mathrm{e}}=T\left(Z_{\mathrm{B}}\right)$ for all points $z>Z_{\mathrm{B}}$. This parcel will also have its ionization fraction fixed to $\phi=\phi\left(T\left(Z_{\mathrm{B}}\right), N\left(Z_{\mathrm{B}}\right)\right)$ for points downstream of $z_{\mathrm{B}}$. The freezing of electron temperature is seen in the spatial profile of a tantalum flow at 275 ns in Figure 5, where the freezing point was assumed to occur at $T_{\mathrm{e}}=0.41 \mathrm{eV}$ at any time. Figure 7 shows the flow characteristics on the $z$ - $t$ plane, and the trajectory of the simple $T_{\mathrm{e}}=0.41 \mathrm{eV}$ freezing transition in this example. Parcels of fluid emerge at $z=0$ and move along characteristic lines. The trajectory of the freezing point divides the $z$ - $t$ plane into regions of equilibrium and nonequilibrium. Figure 8 shows the electron number density at 275 ns corresponding to Figures 5 and 7.

\section{Electron beam MHD interaction}

The Faraday's law shown as equation (1) is found by combining a generalized Ohm's law for the current density exclusive of the electron beam $\mathbf{j}$,

$$
\mathbf{j}=\sigma\left(\mathbf{E}+\mathbf{v} \times \mathbf{B}+\frac{\nabla p_{\mathrm{e}}}{\mathrm{e} n_{\mathrm{e}}}\right),
$$

the electron beam current density source $\mathbf{j}_{0}$,

$$
\mathbf{j}_{0}=\operatorname{curl}\left(\frac{\mathbf{B}_{0}}{\mu}\right)
$$


Ampere's law,

$$
\operatorname{curl}\left(\frac{\mathrm{B}}{\mu}\right)=\mathbf{j}+\mathbf{j}_{0}+\varepsilon \frac{\partial \mathrm{E}}{\partial t}
$$

and Faraday's law

$$
\operatorname{curl}(\mathbf{E})=-\frac{\partial \mathbf{B}}{\partial t}
$$

The electron pressure, number density, and temperature are related by the ideal gas law $p_{\mathrm{e}}=\mathrm{k} n_{\mathrm{e}} T_{\mathrm{e}}$. Terms proportional to $\varepsilon / \sigma$ are neglected, which is to say electromagnetic waves are ignored. The beam is taken as a source of magnetism $\left(\mathrm{B}_{0}\right)$ and heat $\left(\nabla T_{\mathrm{e}}\right)$ within the target plasma, but not of charge. The last assumption must be reevaluated in the case of beam penetration of rarefied, low-conductivity clouds. The general features of the resulting Faraday's law are described in the abstract and first section of this report. This section will address two topics: a justification for this generalized Ohm's law, and an estimate of the magnitude of thermally generated magnetization.

A great deal has been written about the generalized Ohm's law, references [16], [17], [18], [19], and [20] are just a sampling. The analysis here does not approach the depth of the references. My purpose is to show that equation (36) is more than a simple arbitrary choice. The current density sought is

$$
\mathbf{j}=\mathrm{Ze} \Gamma_{+}-\mathrm{e} \Gamma_{\mathrm{e}}=\mathrm{e}\left(\mathrm{Z} n_{+} \mathbf{v}_{+}-n_{\mathrm{e}} \mathbf{v}_{\mathrm{e}}\right) .
$$

The velocities are obtained from the momentum equations for electrons and ions, which both have the form

$$
\rho\left[\frac{\partial \mathbf{v}}{\partial t}+\mathbf{v} \cdot \nabla \mathbf{v}\right]=-\nabla p+\mathrm{q}_{ \pm} n(\mathbf{E}+\mathbf{v} \times \mathbf{B})-\rho v_{\mathrm{N}}\left(\mathbf{v}-\mathbf{v}_{\mathrm{N}}\right)
$$

where $\mathrm{q}_{+}=\mathrm{Ze}, \mathrm{q}_{-}=-\mathrm{e}, v_{\mathrm{N}}$ is the electron-ion collision frequency, $\mathbf{v}_{\mathrm{N}}=\left(\rho_{+} \mathbf{v}_{+}+\rho_{\mathrm{e}} \mathbf{V}_{\mathrm{e}}\right) /\left(\rho_{+}+\rho_{\mathrm{e}}\right)$ is the mass average velocity, and $\rho, p, n$ and $\mathbf{v}$ are distinct for each species. The assumed plasma is fully ionized and inviscid. Each flux vector has the form 


$$
\Gamma=n \mathbf{V}_{\mathrm{N}}+n \Omega_{ \pm}(\mathrm{E}+\mathbf{v} \times \mathbf{B})-\frac{n}{v_{\mathrm{N}}}\left(\frac{\mathcal{D} \mathbf{V}}{\mathcal{D} t}+\frac{\nabla p}{\rho}\right)
$$

where $\Omega_{+}=\mathrm{Ze} /\left(\mathrm{m}_{+} v_{\mathrm{N}}\right)$ and $\Omega_{-}=-\mathrm{e} /\left(\mathrm{m}_{\mathrm{e}} v_{\mathrm{N}}\right)$ are the mobility coefficients, and $\mathcal{D} \mathbf{v} / \mathcal{D} t$ is the substantial, or convective, derivative. The current density is

$$
\begin{aligned}
\mathbf{j}=\rho_{\mathrm{c}} \mathbf{v}_{\mathrm{N}}+\sigma \mathrm{E}+\mathrm{e}\left[\Omega_{+} n_{+} \mathbf{v}_{+}-\Omega_{-} n_{\mathrm{e}} \mathbf{v}_{\mathrm{N}}-\Omega_{-} \frac{\mathrm{m}_{+}}{\mathrm{m}_{\mathrm{e}}} n_{+}\left(\mathbf{v}_{\mathrm{N}}-\mathbf{v}_{+}\right)\right] \times \mathrm{B} \\
+\frac{\mathrm{e}}{v_{\mathrm{N}}}\left\{n_{\mathrm{e}}\left(\frac{\mathcal{D} \mathbf{v}_{\mathrm{e}}}{\mathcal{D} t}+\frac{\nabla p_{\mathrm{e}}}{\rho_{\mathrm{e}}}\right)-\mathrm{Z} n_{+}\left(\frac{\mathcal{D} \mathbf{v}_{+}}{\mathcal{D} t}+\frac{\nabla p_{+}}{\rho_{+}}\right)\right\},
\end{aligned}
$$

where $\rho_{\mathrm{C}}=\mathrm{e}\left(\mathrm{Z} n_{+}-n_{\mathrm{e}}\right)$ is the charge density, and $\sigma=\mathrm{e}\left(n_{+} \Omega_{+}-n_{\mathrm{e}} \Omega_{-}\right)$is the conductivity. Because of the great disparity in the masses of ions and electrons, and because of the comparable magnitudes of their temperatures, $\mathbf{v}_{+} \rightarrow \mathbf{v}_{\mathrm{N}}, \sigma \rightarrow-\mathrm{e} n_{\mathrm{e}} \Omega_{-}$, and the electron pressure gradient emerges as the significant term in the curly braces. The Ohm's law shown as equation (36) emerges from (43) by applying these simplifications and the additional assumption of charge neutrality. The subscript is dropped for the mass average velocity in equation (36).

To estimate the magnitude of thermally generated $\mathbf{B}$, we first express equation (1) in cylindrical coordinates and assume a simplified case where the functional dependencies are $\mathbf{B}=B_{\theta}(r, z, t) \mathbf{i}_{\theta}, \mathbf{B}_{0}=B_{0 \theta}(r, t) \mathbf{i}_{\theta}$, $\mathbf{v}=V_{\mathrm{z}}(z, t) \mathbf{i}_{\mathrm{z}}, n_{\mathrm{e}}(r, z, t), T_{\mathrm{e}}(r, t)$, and $\sigma(r, t)$. In addition, $\sigma(r, t)$ is assumed to be purely dependent on $T_{\mathrm{e}}^{3 / 2}$, so $\partial \sigma / \partial r=(3 / 2)\left(\sigma / T_{\mathrm{e}}\right)\left(\partial T_{\mathrm{e}} / \partial r\right)$. The result is

$$
\begin{aligned}
\frac{\partial B_{\theta}}{\partial t}=\left\{\frac{\partial T_{\mathrm{e}}}{\partial r}[\right. & \left.\left.\frac{-\mathrm{k}}{\mathrm{e} n_{\mathrm{e}}} \frac{\partial n_{\mathrm{e}}}{\partial Z}+\frac{1}{\sigma \mu r}\left(B_{0 \theta}+r \frac{\partial B_{0 \theta}}{\partial r}\right) \frac{3}{2 T_{\mathrm{e}}}\right]-\frac{1}{\sigma \mu r}\left(\frac{\partial B_{0 \theta}}{\partial r}+r \frac{\partial^{2} B_{0 \theta}}{\partial r^{2}}\right)\right\} \\
& -B_{\theta}\left(\frac{\partial V_{\mathrm{Z}}}{\partial Z}+\frac{3}{2} \frac{1}{\sigma \mu r} \frac{1}{T_{\mathrm{e}}} \frac{\partial T_{\mathrm{e}}}{\partial r}\right)-V_{\mathrm{Z}} \frac{\partial B_{\theta}}{\partial Z}+\frac{1}{\sigma \mu} \frac{\partial^{2} B_{\theta}}{\partial Z^{2}} \\
& +\frac{1}{\sigma \mu}\left\{\left(\frac{1}{r}-\frac{3}{2} \frac{1}{T_{\mathrm{e}}} \frac{\partial T_{\mathrm{e}}}{\partial r}\right) \frac{\partial B_{\theta}}{\partial r}+\frac{\partial^{2} B_{\theta}}{\partial r^{2}}\right\} .
\end{aligned}
$$

The radial derivatives will depend on the radial profile of the electron beam, and the axial derivatives will depend on the axial profile of the 
cloud. The following three sections describe separate analyses to find these derivatives, then we return to apply them to equation (44).

\section{Electron beam profile - radial derivatives}

Assume the electron beam has a radial current profile of

$$
I_{0}(r)=\frac{I_{00}}{\left(1+\left(\sqrt{2} \frac{r}{r_{0}}\right)^{2}\right)^{2}}
$$

where $I_{0}(r)$ is the total current included between radius $r$ and infinity. The current density at radius $r$ is

$$
j_{0}(r)=\frac{4 I_{00}}{\pi r_{0}^{2}} \frac{1}{\left(1+2\left(\frac{r}{r_{0}}\right)^{2}\right)^{3}},
$$

and the magnetic induction is

$$
B_{0}(r)=\frac{\mu j_{0}(0) r_{0}}{8}\left(\frac{r_{0}}{r}\right)\left\{1-\frac{1}{\left(1+2\left(\frac{r}{r_{0}}\right)^{2}\right)^{2}}\right\} .
$$

The total electron beam current $I_{00}$ is a function of time. Figure 9 shows the normalized radial profiles for $j_{0}(r)$ and $B_{0}(r)$ along normalized coordinate $X=r / r_{0}$, as well the normalized radial profile for $\partial B_{\theta} / \partial t$, which will be presented later.

The electron temperature at a given $r$ is

$$
T_{\mathrm{e}}(r, t)=\left(\frac{\gamma-1}{\gamma}\right)\left(\frac{\mathrm{m}}{\mathrm{k}}\right)(\Delta E) \int_{0}^{t}\left|j_{0}(r, \tau)\right| d \tau,
$$

where $\Delta E=1.6 \times 10^{5}(\mathrm{eV} / \mathrm{m}) /\left(\mathrm{kg} / \mathrm{m}^{3}\right)$ and is an average energy loss by collisions as a relativistic electron beam penetrates a target cloud of atomic mass $\mathrm{m}$. The electron beam is assumed to be a pulse that is short in comparison to the evolution of the cloud. The radial profile of $T_{\mathrm{e}}$ is the same as that for $j_{0}$. 
The radial derivatives needed in equation (44), which result from this beam profile are:

$$
\begin{gathered}
\frac{1}{T_{\mathrm{e}}} \frac{\partial T_{\mathrm{e}}}{\partial r}=-\left(\frac{12}{r_{0}}\right) \frac{x}{\left(1+2 x^{2}\right)} \\
\frac{\partial B_{0}}{\partial r}=\left(\frac{1}{r_{0}}\right)\left(\frac{\mu j_{0}(0) r_{0}}{8}\right)\left\{\frac{8}{\left(1+2 x^{2}\right)^{3}}-\frac{1}{x^{2}}\left[1-\frac{1}{\left(1+2 x^{2}\right)^{2}}\right]\right\} \\
\frac{\partial^{2} B_{0}}{\partial r^{2}}=\left(\frac{1}{r_{0}^{2}}\right)\left(\frac{\mu j_{0}(0) r_{0}}{8}\right)\left\{\frac{2}{x^{3}}\left[1-\frac{1}{\left(1+2 x^{2}\right)^{2}}\right]-\frac{8}{x} \frac{1}{\left(1+2 x^{2}\right)^{3}}-\frac{96 x}{\left(1+2 x^{2}\right)^{4}}\right\}
\end{gathered}
$$

where $x=r / r_{0}$.

\section{Cloud density - the axial derivatives}

The model of cloud flow, which was described earlier in this report, has an idealized density front of infinite slope. In reality, particles of higher than average temperature will diffuse ahead of the mass-average flow, and particles of lower than average temperature will lag. In this way an initially sharp density front relaxes into a broader zone of lesser gradient. To capture this effect, and to have a differentiable model of the density profile, the following form is used for the axial profile

$$
N(z)=\left(N_{2}-N_{1} \mathrm{e}^{-b_{2} z_{1}}\right)\left(\frac{Z}{Z_{1}}\right)^{b_{1}}+N_{1} \mathrm{e}^{-b_{2} z}-N_{2} \mathrm{e}^{-b_{3}\left(z-z_{1}\right)^{2}},
$$

where $Z<Z_{1}$, which is the coordinate at zero density. This profile has three sections: an exponential decay near the source, a density ramp at midspan in the cloud, and a sharp, gaussian fall-off at the front. Parameters are assigned as follows:

$$
N_{1}=\frac{N_{0}(0)}{\left(1+\frac{t}{t_{0}}\right) \frac{2}{\gamma-1}},
$$




$$
\begin{aligned}
& N_{2}=\frac{N_{0}(0)}{1+\pi\left(\frac{Z}{r_{0}}\right)+2\left(\frac{Z}{r_{0}}\right)^{2}}, \\
& z_{1}=\sqrt{\frac{2}{\gamma-1}} c_{0}(0) t, \\
& b_{1}=t / t_{0}, \\
& b_{2}=1 / r_{0}, \\
& b_{3}=\left(\frac{N_{0}(0) \sigma_{0}}{1+\pi\left(\frac{Z}{r_{0}}\right)+2\left(\frac{z}{r_{0}}\right)^{2}}\right)^{2}
\end{aligned}
$$

Here, $N_{1}$ is the source number density as in equation (6), $N_{2}$ is the density of the idealized sharp front at coordinate $z, z_{1}$ is the position of the sharp front at time $t, b_{1}$ sets the shape of the density ramp behind the front, $b_{2}$ is the scale length of density decay near the source, and $b_{3}$ determines the width of the broadened front at coordinate $z$. The parameters $N_{0}(0), t_{0}, r_{0}$, and $c_{0}(0)$ are as before $\left(N_{0}(0)=\rho(0) / \mathrm{m}\right)$. The atomic cross section is $\sigma_{0}$, and the initial mean-free-path is

$$
\lambda_{0}=1 /\left(N_{0}(0) \sigma_{0}\right) .
$$

A fairly impressive equation results from substituting parameters (53) into equation (52). Figure 10 shows this axial density model for a flow with $t_{0}=93 \mathrm{~ns}, r_{0}=0.4 \mathrm{~mm}, c_{0}=5000 \mathrm{~m} / \mathrm{s}, \lambda_{0}=2 \times 10^{-7} \mathrm{~m}$, and $t$ at $0.2 \mu \mathrm{s}$, $0.4 \mu \mathrm{s}, 0.6 \mu \mathrm{s}, 0.8 \mu \mathrm{s}$, and $1 \mu \mathrm{s}$. Figure 11 shows a second example with $t_{0}=0.5 \mu \mathrm{s}, r_{0}=1.0 \mathrm{~mm}, c_{0}=5000 \mathrm{~m} / \mathrm{s}, \lambda_{0}=2 \times 10^{-7} \mathrm{~m}$, and $t$ at $0.4 \mu \mathrm{s}, 0.8$ $\mu \mathrm{s}, 1.2 \mu \mathrm{s}, 1.6 \mu \mathrm{s}$, and $2 \mu \mathrm{s}$. The profiles in Figures 10 and 11 are normalized by $N_{0}(0)$. The profile of Figure 4 most closely corresponds to the profile at $0.2 \mu \mathrm{s}$ in Figure 10.

The electron density is assumed to have the same axial profile as the flow density. It is important to note that the temperature developed by electron beam heating does not depend on the density of material when the target is thin compared to the electron range. The energy deposited per unit mass is constant, denser matter simply absorbs more energy. The energy per unit mass that is absorbed depends on the atomic weight of the material, so different materials will exhibit different heating from a given beam. 
Because of these effects, the electron temperature only has radial variation, set by the beam profile. The electron density at a given radius is the product of a constant ionization fraction for that radius and the material density. Thus, $\left(1 / n_{\mathrm{e}}\right)\left(\partial n_{\mathrm{e}} / \partial Z\right)=(1 / N)(\partial N / \partial Z)$.

While equations (52) and (53) simplify the task of calculating cloud density profiles, they are still arduous to differentiate. Thus, the axial derivatives will be found for three limiting cases of this density model: $z / z_{1} \rightarrow 0, z / z_{1} \rightarrow 1 / 2, z / z_{1} \rightarrow 1$, which correspond to regions near the source, at midspan, and at the front. It is always assumed that $z_{1}>r_{0}$, and that we consider cross sections at $z>r_{0}$. Implementing these assumptions results in:

$$
\frac{1}{n_{\mathrm{e}}} \frac{\partial n_{\mathrm{e}}}{\partial Z}=-\frac{1}{r_{0}}
$$

near the source,

$$
\frac{1}{n_{\mathrm{e}}} \frac{\partial n_{\mathrm{e}}}{\partial Z}=\frac{1}{Z}\left(\frac{t}{t_{0}}-2\right)
$$

at midspan for a flow at a time $t$ in its evolution, and

$$
\begin{aligned}
& \frac{1}{n_{\mathrm{e}}} \frac{\partial n_{\mathrm{e}}}{\partial z}=-2 \frac{\left(z_{1}-z\right)}{\left(\xi \lambda_{0}\right)^{2}} \frac{\mathrm{e}^{-\left(\frac{z_{1}-z}{\xi \lambda_{0}}\right)^{2}}}{1-\mathrm{e}^{-\left(\frac{z_{1}-z}{\xi \lambda_{0}}\right)^{2}}} \\
& \xi \equiv\left(\frac{4}{\gamma-1}\right)\left(\frac{c_{0}(0) t}{r_{0}}\right)^{2}
\end{aligned}
$$

in the front, which has a density profile

$$
N(z)=\frac{N_{0}(0)}{\xi}\left(1-\mathrm{e}^{-\left(\frac{z_{1}-z}{\xi \lambda_{0}}\right)^{2}}\right) \text {. }
$$

The peak of $\partial^{2} N / \partial z^{2}$ occurs at $\left(z_{1}-z\right) / \xi \lambda_{0}=1 / \sqrt{ } 2$, and the logarithmic derivative there is 


$$
\frac{1}{n_{\mathrm{e}}} \frac{\partial n_{\mathrm{e}}}{\partial Z}=-\frac{2.18}{\xi \lambda_{0}}=-\frac{2.18}{\lambda_{0}}\left(\frac{\gamma-1}{4}\right)\left(\frac{r_{0}}{c_{0}(0) t}\right)^{2}
$$

The derivatives in equation (44) for $\partial B_{\theta} / \partial t$ that remain unspecified are the $z$ derivative of $v_{z}$, and the first and second $r$ and $z$ derivatives of $B_{\theta}$ itself. The following section describes these derivatives.

\section{The variations of the magnetic induction}

The leading term of equation (44) will produce the dominate growth of $B_{\theta}$ when there is an appreciable density gradient. The radial variation of this term is set by the radial gradient of the electron temperature. Thus, the form expected for $B_{\theta}$ is

$$
B_{\theta}(r, z)=b_{\theta}(z) \frac{x}{\left(1+2 x^{2}\right)^{4}} .
$$

For convenience later, define $f_{\mathrm{B}}(x)$ as the radial factor in equation (60); this is shown as curve $" \mathrm{~dB}_{\theta}$ " in Figure 9. The $r$ derivatives of $B_{\theta}$ are:

$$
\begin{aligned}
& \frac{1}{B_{\theta}} \frac{\partial B_{\theta}}{\partial r}=\frac{1}{r_{0} X} \frac{1-14 X^{2}}{1+2 X^{2}} \\
& \frac{1}{B_{\theta}} \frac{\partial^{2} B_{\theta}}{\partial r^{2}}=\frac{-16}{r_{0}^{2}} \frac{3+14 x^{2}}{\left(1+2 x^{2}\right)^{2}}
\end{aligned}
$$

The $z$ variation of $B_{\theta}$ should follow that of the electron density gradient, so the following assumption is made

$$
\frac{1}{B_{\theta}} \frac{\partial^{\mathrm{n}} B_{\theta}}{\partial Z^{\mathrm{n}}}=\left(\frac{1}{n_{\mathrm{e}}} \frac{\partial n_{\mathrm{e}}}{\partial Z}\right)^{\mathrm{n}}(-1)^{\mathrm{n}-1} .
$$

The $z$ variation of $v_{Z}$ is assumed to have little impact as it is quite gradual. Now we return to equation (44). 


\section{The growth of magnetic induction during a heating pulse}

Applying the results described in the last three sections to equation (44) produces

$$
\begin{aligned}
\frac{\partial B_{\theta}}{\partial t}= & -\left(\frac{\mathrm{k} T_{\mathrm{e}}}{\mathrm{e}}\right)\left(\frac{1}{T_{\mathrm{e}}} \frac{\partial T_{\mathrm{e}}}{\partial r}\right)\left(\frac{1}{n_{\mathrm{e}}} \frac{\partial n_{\mathrm{e}}}{\partial Z}\right) \\
& -\left(\frac{1}{\sigma \mu r_{0}^{2}}\right)\left(\frac{\mu j_{0}(0) r_{0}}{8}\right)\left\{\frac{96 x}{\left(1+2 x^{2}\right)^{4}}+\frac{1}{x^{2}}\left[1-\frac{1}{\left(1+2 x^{2}\right)^{2}}\right]\right\} \\
& +\left\{( \frac { 1 } { \sigma \mu r _ { 0 } ^ { 2 } } ) \left[\frac{12}{1+2 x^{2}}+\left(1+\frac{18 x^{2}}{1+2 x^{2}}\right) \frac{1}{x^{2}}\left(\frac{1-14 x^{2}}{1+2 x^{2}}-16 \frac{3+14 x^{2}}{1+2 x^{2}}\right]\right.\right. \\
& \left.+\left(\frac{1}{n_{\mathrm{e}}} \frac{\partial n_{\mathrm{e}}}{\partial Z}\right)\left[\frac{-1}{\sigma \mu}\left(\frac{1}{n_{\mathrm{e}}} \frac{\partial n_{\mathrm{e}}}{\partial Z}\right)-V_{\mathrm{z}}\right]-\frac{\partial V_{\mathrm{z}}}{\partial Z}\right\} B_{\theta},
\end{aligned}
$$

which has the form

$$
\frac{\partial B_{\theta}}{\partial t}=K(r, z, t)+\omega(r, z, t) B_{\theta} .
$$

The source term $K$ includes the effects of thermodynamics and the beam magnetization. The relaxation rate $\omega$ includes the effects of diffusion and transport. For convenience later, define $f_{\mathrm{K}}(x)$ as the factor within the curly braces in the second line of equation (63), and define $f_{\omega}(x)$ as the first square-bracketed factor within the curly braces that span lines three and four of equation (63).

When the electron beam current has a linear growth with time, then by equation (48) the electron temperature increases as time squared. Assume this is the case, and that the following apply:

$$
\begin{aligned}
& K(r, z, t)=t K_{1}(r, z)+t^{2} K_{2}(r, z), \\
& K(r, z, 0)=0, \\
& \omega(r, z, t) \rightarrow \omega(r, z) .
\end{aligned}
$$


The result for $B_{\theta}$ is

$$
B_{\theta}=\frac{K_{1}}{\omega^{2}}\left(\mathrm{e}^{\omega t}-1-\omega t\right)+\frac{K_{2}}{\omega^{3}}\left(2 \mathrm{e}^{\omega t}-1-(1+\omega t)^{2}\right) .
$$

This linear beam is described as follows:

$$
\begin{aligned}
& j_{0}(0, t)=j_{\infty} \frac{t}{t_{\infty}}, \\
& B_{0}\left(0.816 r_{0}, t\right)=\frac{\mu j_{\infty} r_{0}}{8} \frac{t}{t_{\infty}}, \\
& \frac{\mathrm{k} T_{\mathrm{e}}(0, t)}{\mathrm{e}}=\frac{\gamma-1}{\gamma} \frac{\mathrm{m}}{\mathrm{e}} \Delta E \frac{\left|j_{\infty}\right| t_{\infty}}{2}\left(\frac{t}{t_{\infty}}\right)^{2},
\end{aligned}
$$

where the subscript $\infty$ indicates quantities at the end of the beam ramp. Now, find $K_{1}, K_{2}$, and $\omega$ for this linear beam,

$$
\begin{aligned}
& K_{1}=-\frac{B_{0}\left(0.816 r_{0}, t_{\infty}\right)}{t_{\infty}} \frac{f_{\mathrm{K}}(x)}{\sigma \mu r_{0}^{2}}, \\
& K_{2}=\frac{\mathrm{k} T_{\mathrm{e}}\left(0, t_{\infty}\right)}{\mathrm{e}} \frac{1}{t_{\infty}^{2}} \frac{12}{r_{0}} f_{\mathrm{B}}(x)\left(\frac{1}{n_{\mathrm{e}}} \frac{\partial n_{\mathrm{e}}}{\partial Z}\right), \\
& \omega=\left(\frac{1}{n_{\mathrm{e}}} \frac{\partial n_{\mathrm{e}}}{\partial Z}\right)\left[\frac{-1}{\sigma \mu}\left(\frac{1}{n_{\mathrm{e}}} \frac{\partial n_{\mathrm{e}}}{\partial Z}\right)-V_{\mathrm{Z}}\right]-\frac{\partial V_{\mathrm{Z}}}{\partial Z}+\frac{f_{\omega}(x)}{\sigma \mu r_{0}^{2}} .
\end{aligned}
$$

$K_{1}$ retains the form shown in (68), near the source, at midspan, and at the front of the beam. This source factor arises from the beam magnetization. Three forms for each of $K_{2}$ and $\omega$, for the three sections of the cloud, are given by applying logarithmic derivatives (55), (56), and (59) in (68).

If we consider just the front, with an overwhelming gradient effect, then

$$
K_{2}=-\frac{\mathrm{k} T_{\mathrm{e}}\left(0, t_{\infty}\right)}{\mathrm{e}} \frac{1}{t_{\infty}^{2}} \frac{12}{r_{0}} f_{\mathrm{B}}(x) \frac{2.18}{\lambda_{0}}\left(\frac{\gamma-1}{4}\right)\left(\frac{r_{0}}{c_{0}(0) \tau}\right)^{2},
$$




$$
\omega=\frac{-1}{\sigma \mu}\left(\frac{2.18}{\lambda_{0}}\left(\frac{\gamma-1}{4}\right)\left(\frac{r_{0}}{c_{0}(0) \tau}\right)^{2}\right)^{2},
$$

where $\tau$ is used to indicate the elapsed time of the flow to avoid confusion with $t$, which is now used to indicate time in the heating pulse. $K_{1}$ in the front is given by (68), and $B_{\theta}$ by (66).

Figure 12 shows an example of a linear beam with $I_{0}\left(0, t_{\infty}\right)=2000 \mathrm{~A}$, $r_{0}=0.5 \mathrm{~mm}, t_{\infty}=20 \mathrm{~ns}$, and $B_{0}\left(0.816 r_{0}, t_{\infty}\right)=0.8 \mathrm{~T}$, which pierces a front with $c_{0}(0)=5000 \mathrm{~m} / \mathrm{s}, \tau=1 \mu \mathrm{s}, \lambda_{0}=2 \times 10^{-7} \mathrm{~m}$ and $\sigma=10^{6} \mathrm{~S} / \mathrm{m}$. The values used in the radial functions are $f_{\mathrm{K}}(0.3)=17.99$ and $f_{\mathrm{B}}(0.3)=0.1547$. The relaxation rate given by (69) is $-1 / \omega=3.81 \mathrm{~ns}$. The figure shows four magnetic induction histories during the course of the 20 ns pulse, and then again during the initial $2 \mathrm{~ns}$. $B_{\theta}$ is the total induction given by equation (66) for this example, $B_{\theta 1}$ is that part of $B_{\theta}$ generated by $K_{1}$, and $B_{\theta 2}$ is generated by $K_{2}$. $B_{0}$ is the magnetic induction of the beam. The generated fields are shown with reverse polarity so as to compare with the magnitude of the beam induction. Initially, the front magnetizes as a result of eddy currents arising to shield the cloud from the penetration of $B_{0}$. However, this is overtaken by thermal magnetization by 2 ns. After 10 ns, the thermal magnetization completely dominates, and by the end of the $20 \mathrm{~ns}$ ramp it is three times stronger than the beam induction.

A self-consistent treatment of $\sigma\left[T_{\mathrm{e}}(r, t)\right]$ would improve the estimate of $B_{\theta}(r, z, t)$, however that is left to future work. Another consideration is that any estimate of $B_{\theta}$ always remain within the bounds set by the available heat energy density, $B_{\theta}^{2} /(2 \mu)<p_{\mathrm{e}}=\mathrm{k} n_{\mathrm{e}} T_{\mathrm{e}}$, (or e $n_{\mathrm{e}} T_{\mathrm{e}}$ for $T_{\mathrm{e}}$ in $\left.\mathrm{eV}\right)$. The only effects limiting the growth of $B_{\theta}$ in equation (63) are transport and diffusion. An Ohm's law more detailed than equation (36) would offer more avenues for magnetic field saturation, examples are described by Haines [20]. Note that a 1 Tesla field has an energy density of $4 \times 10^{5} \mathrm{~J} / \mathrm{m}^{3}$, and a plasma at $T_{\mathrm{e}}=10 \mathrm{eV}$ and $n_{\mathrm{e}}=2.5 \times 10^{19} \mathrm{~cm}^{-3}$ has one hundred times more at $4 \times 10^{7} \mathrm{~J} / \mathrm{m}^{3}$. The electron beam plasmas described in this report are amply heated to generate magnetic defocusing. 


\section{Deflection of beam electrons}

Clouds with density profiles that increase monotonically toward the source would have the greatest defocusing reaction to an electron beam. Consider two examples where a pair of electron beam pulses hit tantalum targets. The first pulse generates a cloud, and the second pulse magnetizes it. The first example has a $2 \mathrm{~mm}$ thick target plate and pulses separated by $\tau=t_{0}=0.934 \mu \mathrm{s}$. This example is actually \#3 in our series of axial density models. Example 4 has a $1 \mathrm{~mm}$ thick tantalum target and pulses separated by $\tau=t_{0}=0.467 \mu \mathrm{s}$. Recall that $\tau$ is the elapsed time of the flow, and $t_{0}$ is the characteristic time of the source. Clouds at $\tau=t_{0}$ have a negative density gradient by equation (56). The electron beam pulses have $I_{0}\left(0, t_{\infty}\right)=6000 \mathrm{~A}, r_{0}=0.5 \mathrm{~mm}, t_{\infty}=20 \mathrm{~ns}, B_{0}\left(0.816 r_{0}, t_{\infty}\right)=2.4 \mathrm{~T}$, and $\mathrm{a}$ total charge $Q_{0}=I_{0}\left(0, t_{\infty}\right) \Delta t$, where $\Delta t=40$ ns (the equivalent square pulse). In both of these examples, the initial source temperature is $T_{0}(0)=37 \mathrm{eV}$, and the initial sonic speed is $c_{0}(0)=5709 \mathrm{~m} / \mathrm{s}$. The initial front thickness assumed is $\lambda_{0}=2 \times 10^{-6} \mathrm{~m}$, and the conductivity assumed is $\sigma=10^{6} \mathrm{~S} / \mathrm{m}$. The peak temperature in the cloud during the ramp up of beam current is $T_{\mathrm{e}}\left(0, t_{\infty}\right)=37 \mathrm{eV}$, on axis at $t_{\infty}$.

Figure 13 shows the axial density profile for example 3 by equations (52), (53), and (54). Figure 14 shows the axial profile of magnetic induction for this density profile by equations (68), with $V_{\mathrm{Z}}$ and $\partial V_{\mathrm{Z}} / \partial Z$ ignored, and $f_{\omega}(0.3)=-53.4$. The relaxation rate $\omega(r, z)$ in these examples is dominated by the diffusion term with $f_{\omega}$. Figure 15 shows a similar $B_{\theta}\left(0.3 r_{0}, z, t_{\infty}\right)$ profile for the cloud from the $1 \mathrm{~mm}$ plate. The magnetic induction profiles show plateaus below $Z=r_{0}$ where the logarithmic derivatives of density are assumed to be constant at $-1 / r_{0}$, and they show spikes at the fronts. Notice that the magnetic induction within the cloud has a comparable magnitude to the vacuum induction of the beam but is of opposite polarity.

The deflection of beam electrons by $B_{\theta}\left(0.3 r_{0}, z, t_{\infty}\right)$ is estimated by finding the electron gyroradius based on the average of $B_{\theta}$ along $z$ in the cloud, and then the lateral excursion $\Delta y$ for a swing of axial extent $\Delta z$, for the length of the cloud. A small study of examples 3 and 4 yields the following table. 


\section{Table, Deflection of beam electrons}

\begin{tabular}{|c|c|c|c|c|c|c|}
\hline plate & \multicolumn{3}{|c|}{$2 \mathrm{~mm}$--------------------> } & \multicolumn{3}{|c|}{$1 \mathrm{~mm} \quad-------------->$} \\
\hline$B_{\theta}, \mathrm{T}$ & $-0.79 \mathrm{~T}, \mathrm{a}$ & verage & $--->$ & -0.92 & $\mathrm{~T}$, average & $--->$ \\
\hline$\Delta Z, \mathrm{~mm}$ & $8.43 \mathrm{~mm}$ & ------- & $---->$ & 4.22 & $\mathrm{~mm}$ & $--->$ \\
\hline $\mathrm{MeV}$ & 5 & 10 & 20 & 5 & 10 & 20 \\
\hline$\Delta y, \mathrm{~mm}$ & 1.58 & 0.81 & 0.41 & 0.45 & 0.24 & 0.12 \\
\hline$\Delta \theta,^{\circ}$ & 21.3 & 11.0 & 5.6 & 12.2 & 6.4 & 3.3 \\
\hline$\left(\underline{\Delta y+r_{0}}\right)$ & 4.17 & 2.62 & 1.82 & 1.90 & 1.47 & 1.24 \\
\hline $\begin{array}{c}r_{0} \\
\frac{r_{0}}{\left(\Delta y+r_{0}\right)^{2}}\end{array}$ & 0.06 & 0.15 & 0.30 & 0.28 & 0.46 & 0.65 \\
\hline
\end{tabular}

Electrons of a given energy enter the cloud at $0^{\circ}$ and experience a deflection $\Delta y$, and a veering to $\Delta \theta$, after traversing an extent $\Delta z$. These pulses expand noticeably within 20 ns of their arrival at the cloud fronts; the penultimate line in the table shows the growth ratio of the beam diameter, and the last line shows the corresponding intensity diminution. Electron-atom scattering would add to the broadening of the beam.

\section{At the mercy of parameters}

Clearly, any physics conclusion made on the basis of formulas, such as those described in this report, will be at the mercy of the parameters one chooses. For example, the parameter $\lambda_{0}$, which characterizes the initial thickness of the vacuum-to-metal interface, has a major impact on thermal magnetization at the cloud front. Other key parameters are the initial temperature of the fluid source, and the conductivity of the cloud. The model invites one to play with parametric assumptions, and it has value to the extent that this produces insight. 


\section{Acknowledgment}

I would like to acknowledge Professor Malcolm Haines, of Imperial College, London, for his encouragement and his patience in answering my questions during his visit to LLNL in the summer of 1998.

\section{References}

1. M. Garcia, "Splash flow from a metal plate hit by an intense electron beam pulse," Lawrence Livermore National Laboratory, UCRL-ID128660, 4 September 1997.

2. M. Garcia, "Frozen plasma within the flow from a metal plate hit by an electron beam pulse," Lawrence Livermore National Laboratory, UCRL-ID-126296, 12 November 1997.

3. M. Garcia, "On electromagnetic acceleration of material from a plate hit by a pulsed electron beam," Lawrence Livermore National Laboratory, UCRL-JC-130448, 16 April 1998.

4. M. Garcia, "Electron beam expansion by target heating," Lawrence Livermore National Laboratory, UCRL-ID-131291, 2 July 1998.

5. M. Garcia, "Self-effects in expanding electron beam plasmas," in Engineering Research, Development and Technology, FY98, Lawrence Livermore National Laboratory, UCRL-53868-98, 1999.

6. M. Garcia, "Creating metallic under-dense radiators by electron beam heating prior to laser impact," Lawrence Livermore National Laboratory, UCRL-ID-1?????, 15 December 1998.

7. Equations, Tables, and Charts for Compressible Flow, NACA Report 1135, NASA Ames Research Staff, 1953.

8. A. H. Shapiro, The Dynamics and Thermodynamics of Compressible Fluid Flow, Volume 1, New York: The Ronald Press Company, 1953. 
9. Y. B. Zel'dovich and Y. P. Raizer, Physics of Shock Waves and HighTemperature Hydrodynamic Phenomena, in two volumes, W. D. Hayes and R. F. Probstein, editors, New York: Academic Press, 1966 and 1967. See:

1) Volume 1 , chapter 1 , section 28, "Sudden isentropic expansion of a spherical gas cloud into vacuum," section 29, "Conditions for self-similar sudden expansion of a gas cloud into vacuum."

2) Volume 2, chapter 8, topic 3 "Disturbance of thermodynamic equilibrium in the sudden expansion of a gas into vacuum," section 6, "Sudden expansion of a gas cloud," section 7, "Freezing effect," section 8, "Disturbance of ionization equilibrium," section 9, "The kinetics of recombination and cooling of the gas following the disturbance of ionization equilibrium."

10. K. N. C. Bray, "Atomic Recombination in a Hypersonic Wind-Tunnel Nozzle,” Journal of Fluid Mechanics, Vol. 6, pt. 1, p. 1, 1959.

11. K. N. C. Bray, "Electron-Ion Recombination in Argon Flowing through a Supersonic Nozzle," in The High Temperature Aspects of Hypersonic Flow, W. C. Nelson, editor, New York: Macmillan, 1964.

12. W. G. Vincenti and C. H. Kruger, Jr., Introduction to Physical Gas Dynamics, New York: John Wiley and Sons, Inc., 1965.

13. B. G. DeVolder, T. J. T. Kwan, K. D. McLenithan, "Modeling plasma formation in a solid target heated by a multi-MeV electron beam," the 25th IEEE International Conference on Plasma Science, 4C01, 1-4 June 1998, Raleigh, NC.

14. B. G. DeVolder, "The ionization state and electron density of a target plasma generated in the Integrated Test Stand (electron beam facility)," Los Alamos National Laboratory, Research Note X-RN(U)98-033(U), 19 August 1998.

15. J. D. Cobine, Gaseous Conductors, New York: Dover Publications, Inc., 1958.

16. B. S. Tanenbaum, Plasma Physics, New York: McGraw-Hill Book Co., 1967. 
17. M. Mitchner and C. H. Kruger, Jr., Partially Ionized Gases, New York: John Wiley \& Sons, Inc., 1973.

18. S. I. Braginskii, "Transport processes in a plasma," in Reviews of Plasma Physics, M. A. Leontovich, editor, New York: Consultants Bureau, 1965.

19. M. G. Haines, "Magnetic-field generation in laser fusion and hotelectron transport," Canadian Journal of Physics, Vol. 64, 1986, pages 912-919.

20. M. G. Haines, "Saturation mechanisms for the generated magnetic field in nonuniform laser-matter irradiation," Physical Review Letters, Vol. 78, No. 2, 13 January 1997, pages 254-257.

\section{Figure captions}

1. Source number density history. Tantalum number density in $\mathrm{cm}^{-3}$ versus time in $\mu$ s for an impulse of $10 \mathrm{~J}$ in a $0.8 \mathrm{~mm}$ diameter, 100 $\mu \mathrm{m}$ long volume. The parameter $t_{0}=93 \mathrm{~ns}$.

2. Source temperature history. The source temperature in $\mathrm{eV}$ corresponding to Figure 1.

3. Flow fronts. This schematic shows the assumed shape of flow fronts, and described by $A(z)$ in equation (8).

4. Number density profile at $275 \mathrm{~ns}$. Tantalum number density in $\mathrm{cm}^{-3}$ versus axial coordinate $z$ in $\mathrm{mm}$ at $275 \mathrm{~ns}$. The source of this flow is described in Figures 1 and 2. 
5. Temperature profiles at $275 \mathrm{~ns}$. Temperature in eV versus axial coordinate $z$ in $\mathrm{mm}$ at $275 \mathrm{~ns}$. Electron temperature diverges from tantalum fluid temperature in regions of nonequilibrium. This is the same flow as in Figure 4. A static background gas of $10^{-6}$ torr and $300{ }^{\circ} \mathrm{K}$ was assumed to exist in the space outside the source solely for convenience when making logarithmic plots.

6. Velocity profile at $275 \mathrm{~ns}$, and $M(z)$. Velocity in $\mathrm{m} / \mathrm{s}$ versus axial coordinate $z$ in $\mathrm{mm}$ at $275 \mathrm{~ns}$. Also shown is the Mach number profile for flow fronts as described by $A(z)$ in equation (8). This is the flow of Figure 4.

7. Flow characteristics and the freezing line. The $z$ - $t$ plane, in $m m-\mu s$, for the flow of Figure 4. Fluid emerges at $z=0$ over time and propagates along characteristics through an evolving ionizationfreezing transition point. Here, the freezing condition is $T=0.41 \mathrm{eV}$. Frozen flow exists above the freezing line, equilibrium flow below it. The characteristics are curved because the flow accelerates from the source, and they are splayed rather then parallel because the source cools rapidly.

8. Electron density profile at $275 \mathrm{~ns}$. Electron number density in $\mathrm{cm}^{-3}$ versus axial coordinate $z$ in $\mathrm{mm}$ at $275 \mathrm{~ns}$ for the flow of Figure 4. Notice from Figures 5 and 7 that this profile cuts through the freezing line at $0.8 \mathrm{~mm}$. The leading edge of the flow, emanating from a hotter source than later parcels, takes longer to cool to the freezing condition. Later flow starts cooler and reaches frozen ionization sooner. The ionization fraction is about $10^{-4}$ in the frozen flow.

9. Radial profiles set by electron beam. Normalized radial profiles for $j_{0}(r)$ and $B_{0}(r)$ along normalized coordinate $x=r / r_{0}$, as well the normalized radial profile for $\partial B_{\theta} / \partial t$.

10. Axial density model, example 1 . This flow has $t_{0}=93 \mathrm{~ns}, r_{0}=0.4 \mathrm{~mm}$, $c_{0}=5000 \mathrm{~m} / \mathrm{s}, \lambda_{0}=2 \times 10^{-7} \mathrm{~m}$, and $t$ at $0.2 \mu \mathrm{s}, 0.4 \mu \mathrm{s}, 0.6 \mu \mathrm{s}, 0.8 \mu \mathrm{s}$, and $1 \mu \mathrm{s}$.

11. Axial density model, example 2. This flow has $t_{0}=0.5 \mu \mathrm{s}, r_{0}=1 \mathrm{~mm}$, $c_{0}=5000 \mathrm{~m} / \mathrm{s}, \lambda_{0}=2 \times 10^{-7} \mathrm{~m}$, and $t$ at $0.4 \mu \mathrm{s}, 0.8 \mu \mathrm{s}, 1.2 \mu \mathrm{s}, 1.6 \mu \mathrm{s}$, and $2 \mu \mathrm{s}$. 
12. Magnetic field growth during a heating pulse. This is an example of a linear beam with $I_{0}\left(0, t_{\infty}\right)=2000 \mathrm{~A}, r_{0}=0.5 \mathrm{~mm}, t_{\infty}=20 \mathrm{~ns}$, and $B_{0}\left(0.816 r_{0}, t_{\infty}\right)=0.8 \mathrm{~T}$, which pierces a front with $c_{0}(0)=5000 \mathrm{~m} / \mathrm{s}$, $\tau=1 \mu \mathrm{s}, \lambda_{0}=2 \times 10^{-7} \mathrm{~m}$ and $\sigma=10^{6} \mathrm{~S} / \mathrm{m}$. The values used in the radial functions are $f_{\mathrm{K}}(0.3)=17.99$ and $f_{\mathrm{B}}(0.3)=0.1547$. The relaxation rate given by (69) is $-1 / \omega=3.81 \mathrm{~ns}$. The figure shows four magnetic induction histories during the course of the $20 \mathrm{~ns}$ pulse, and then again during the initial 2 ns. $B_{\theta}$ is the total induction given by equation (66) for this example, $B_{\theta 1}$ is that part of $B_{\theta}$ generated by $K_{1}$, and $B_{\theta 2}$ is generated by $K_{2} . B_{0}$ is the magnetic induction of the beam. The generated fields are shown with reverse polarity so as to compare with the magnitude of the beam induction.

13. Axial density model, example 3. This example has a $2 \mathrm{~mm}$ thick tantalum target plate and pulses separated by $\tau=t_{0}=0.934 \mu \mathrm{s}$. The electron beam pulses have $I_{0}\left(0, t_{\infty}\right)=6000 \mathrm{~A}, r_{0}=0.5 \mathrm{~mm}, t_{\infty}=20 \mathrm{~ns}$, $B_{0}\left(0.816 r_{0}, t_{\infty}\right)=2.4 \mathrm{~T}$, and a total charge $Q_{0}=I_{0}\left(0, t_{\infty}\right) \Delta t$, where $\Delta t=40 \mathrm{~ns}$. The initial source temperature is $T_{0}(0)=37 \mathrm{eV}$, and the initial sonic speed is $c_{0}(0)=5709 \mathrm{~m} / \mathrm{s}$. The initial front thickness is $\lambda_{0}=2 \times 10^{-6} \mathrm{~m}$, and the conductivity is $\sigma=10^{6} \mathrm{~S} / \mathrm{m}$. The peak temperature on axis at $t_{\infty}$ in the cloud is $T_{\mathrm{e}}\left(0, t_{\infty}\right)=37 \mathrm{eV}$. The normalized density profiles for example 4 are quite similar.

14. Magnetic induction at $20 \mathrm{~ns}$ in example 3 . This is $B_{\theta}\left(0.3 r_{0}, z, t_{\infty}\right)$ generated in the cloud from the $2 \mathrm{~mm}$ plate, described in Figure 13. The magnetic induction profile has a plateau below $Z=r_{0}$ where the logarithmic derivative of density is assumed to be constant at $-1 / r_{0}$, the spike is at the front.

15. Magnetic induction at $20 \mathrm{~ns}$ in example 4. This is $B_{\theta}\left(0.3 r_{0}, z, t_{\infty}\right)$ generated in the cloud from the $1 \mathrm{~mm}$ plate. Other features are similar to those described in Figures 13 and 14. 


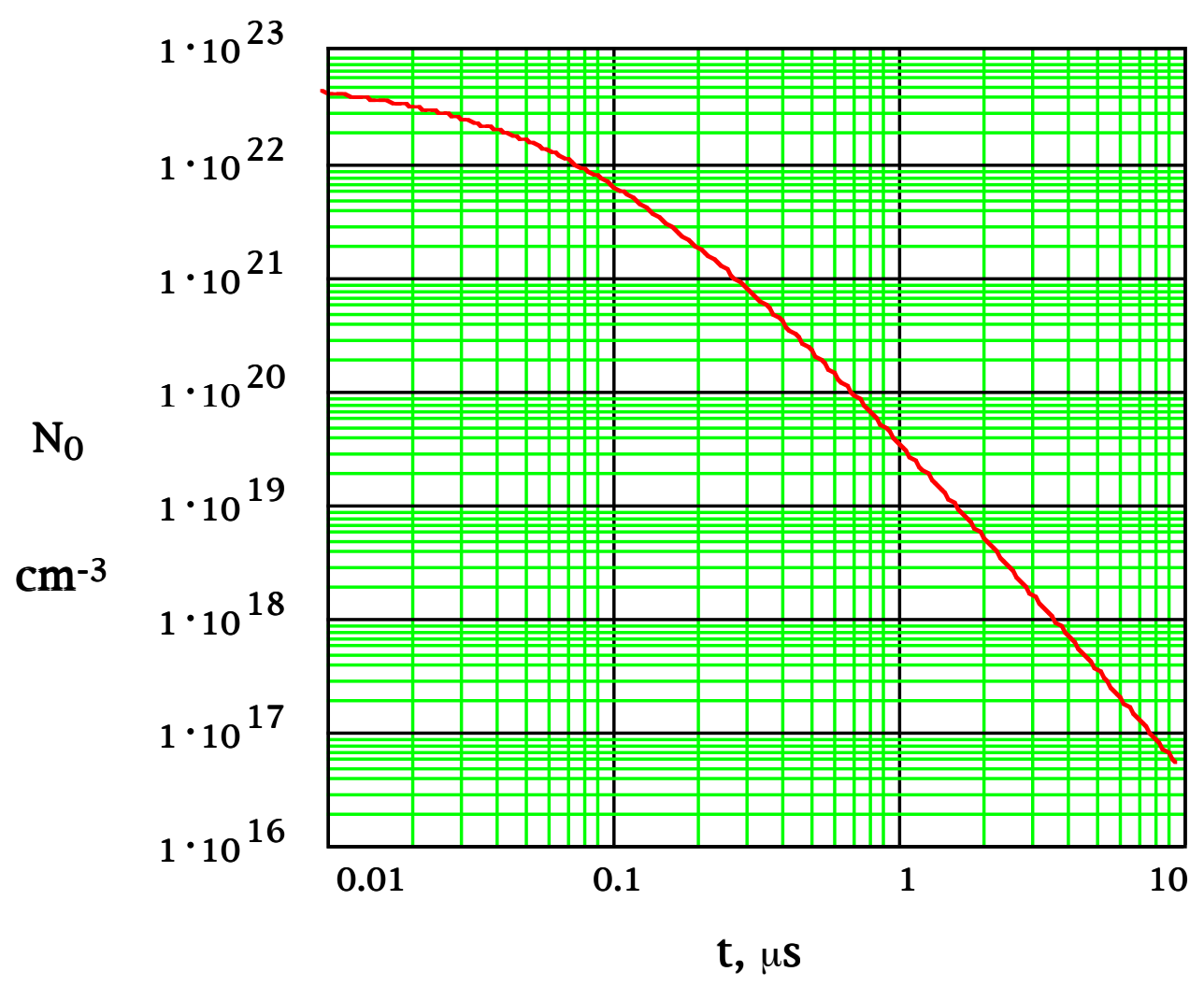

Figure 1, Source number density history 


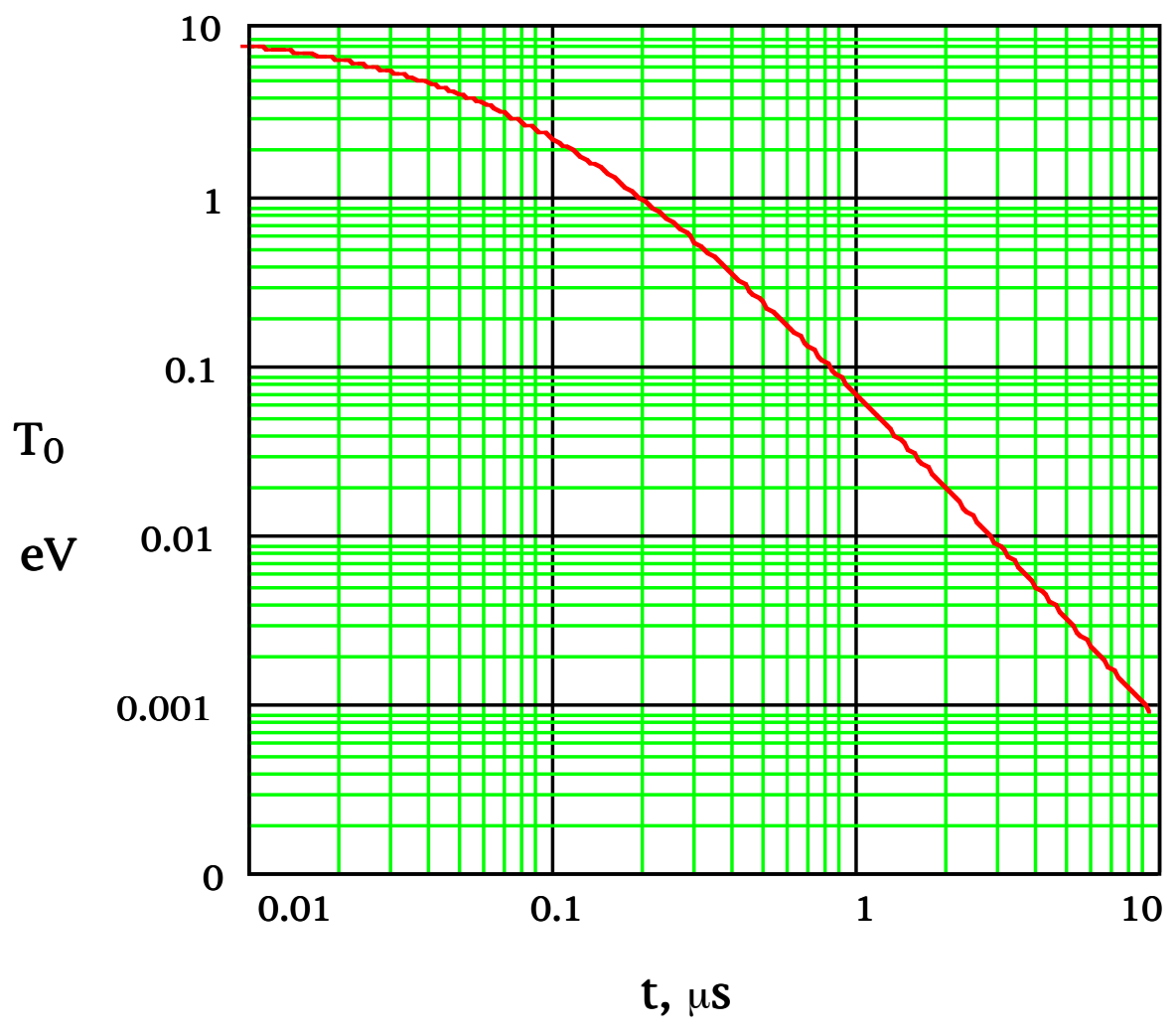

Figure 2, Source temperature history 


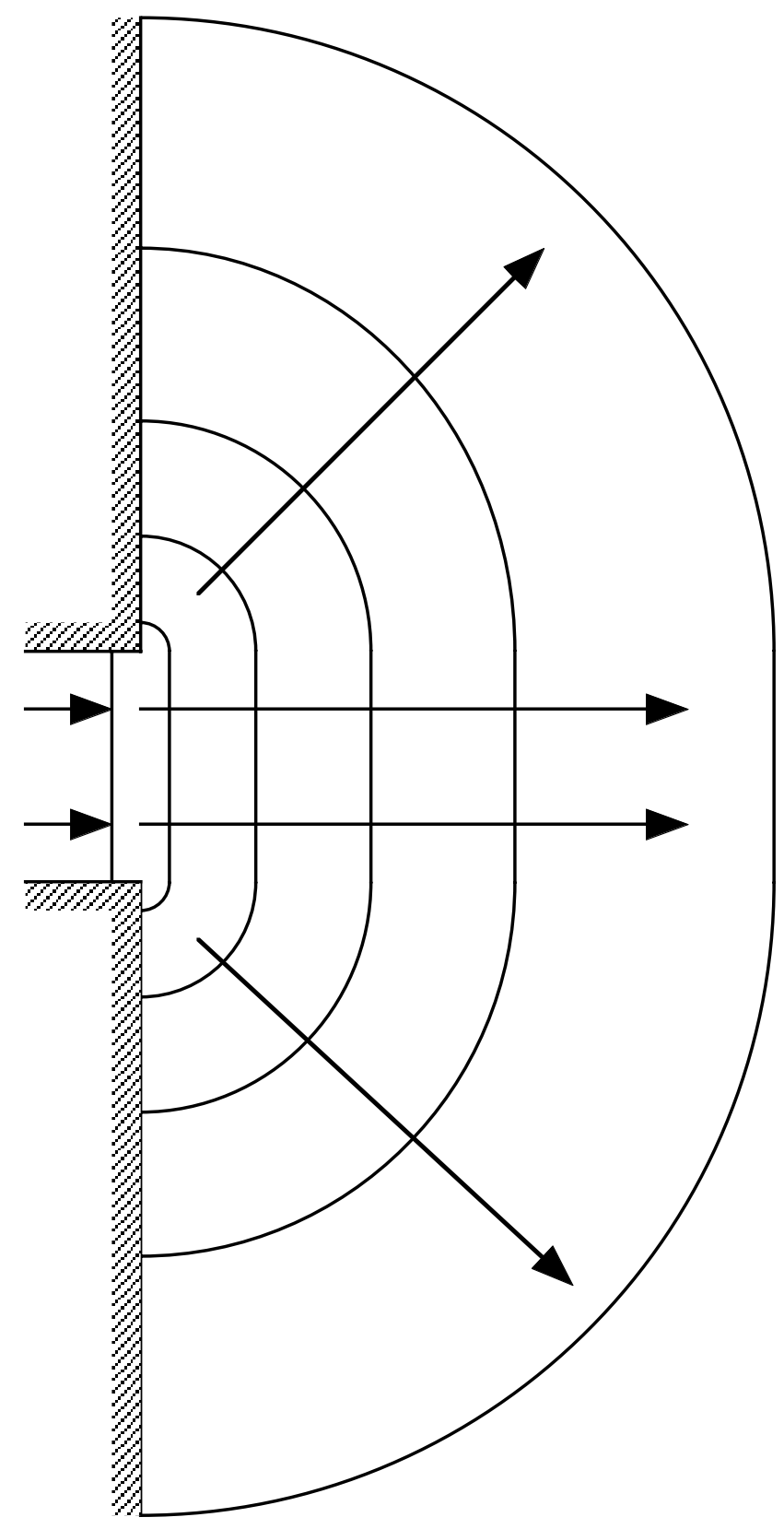

Figure 3, Flow fronts 


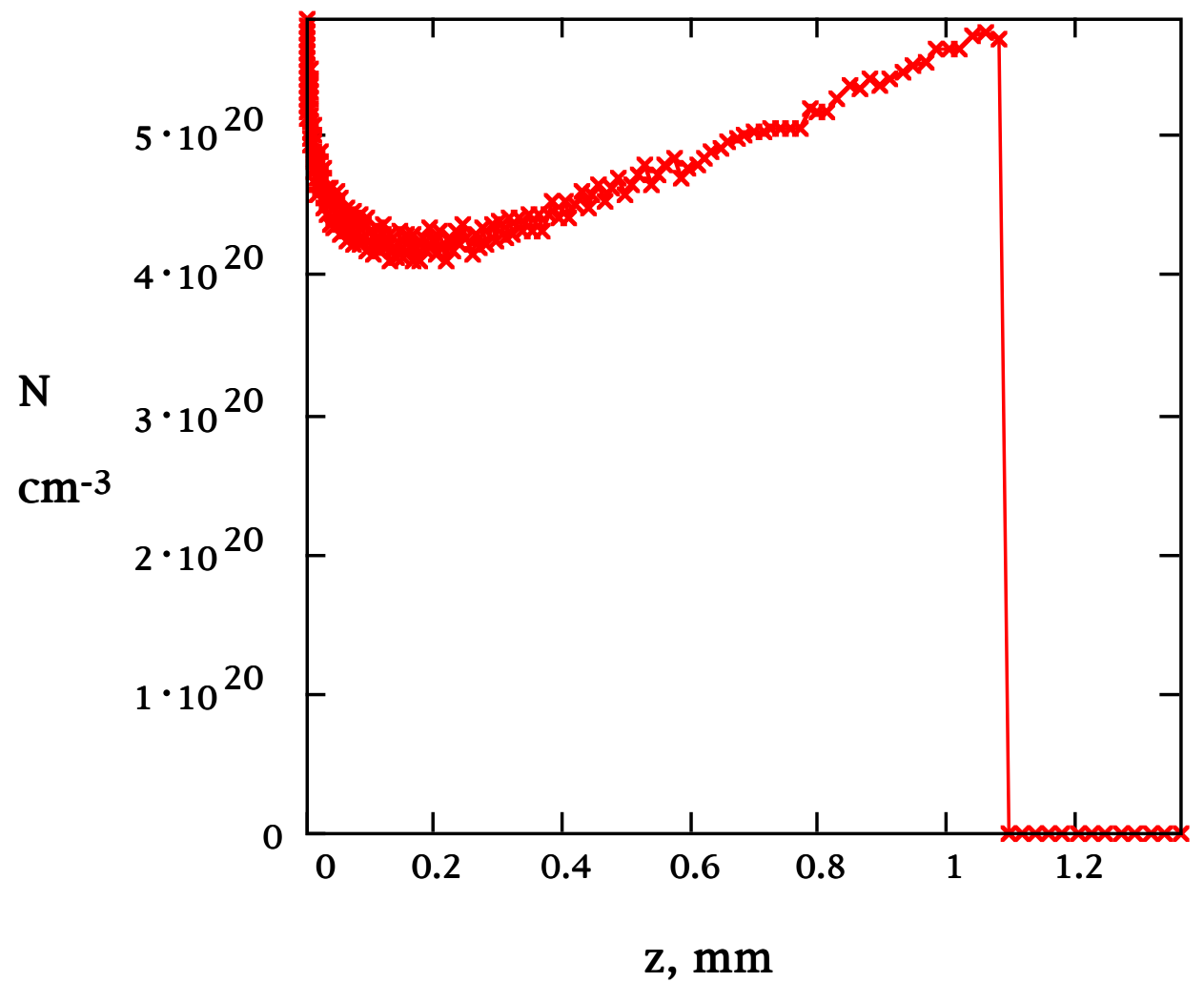

Figure 4, Number density profile at $275 \mathrm{~ns}$ 


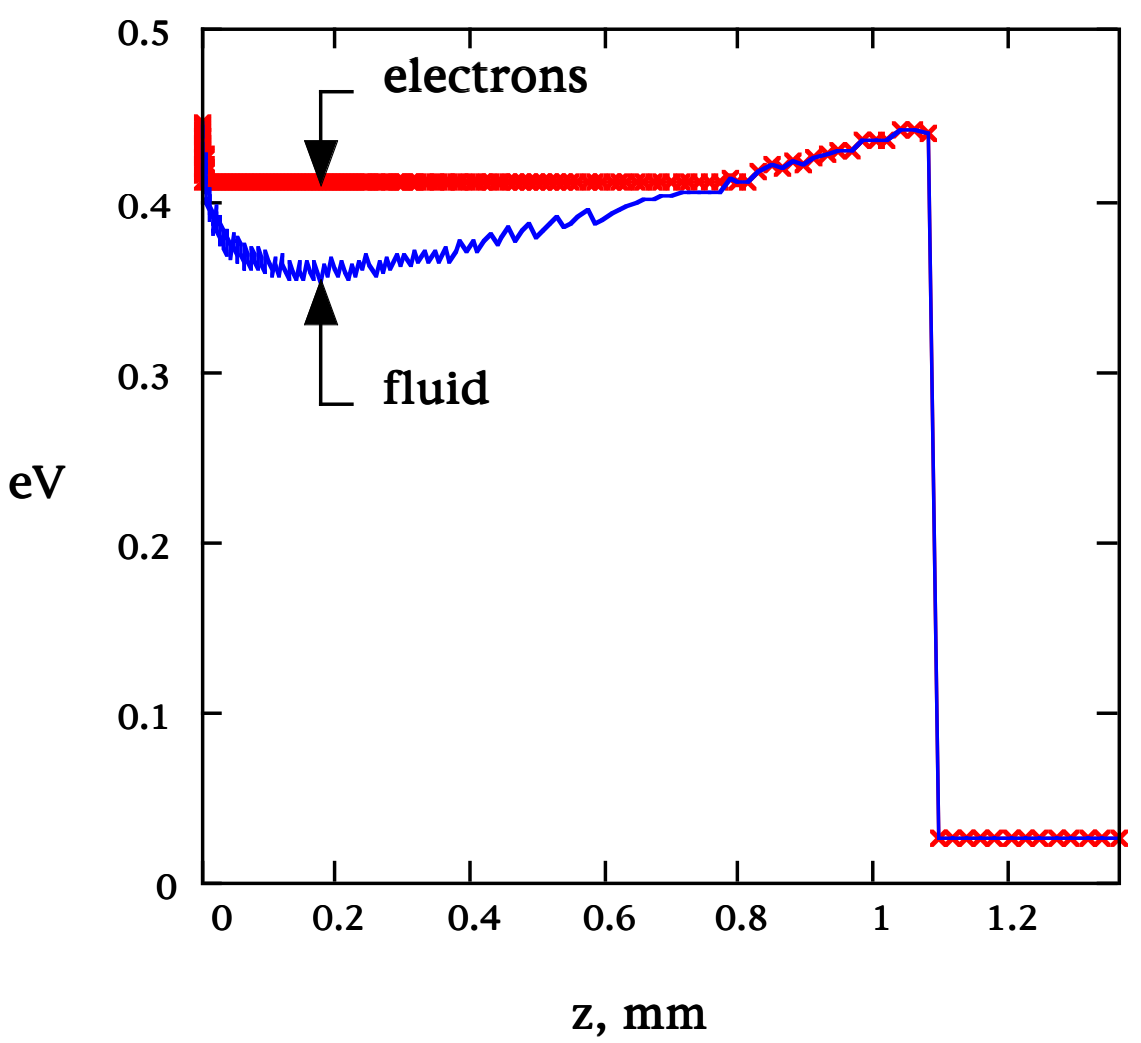

Figure 5, Temperature profiles at $275 \mathrm{~ns}$ 

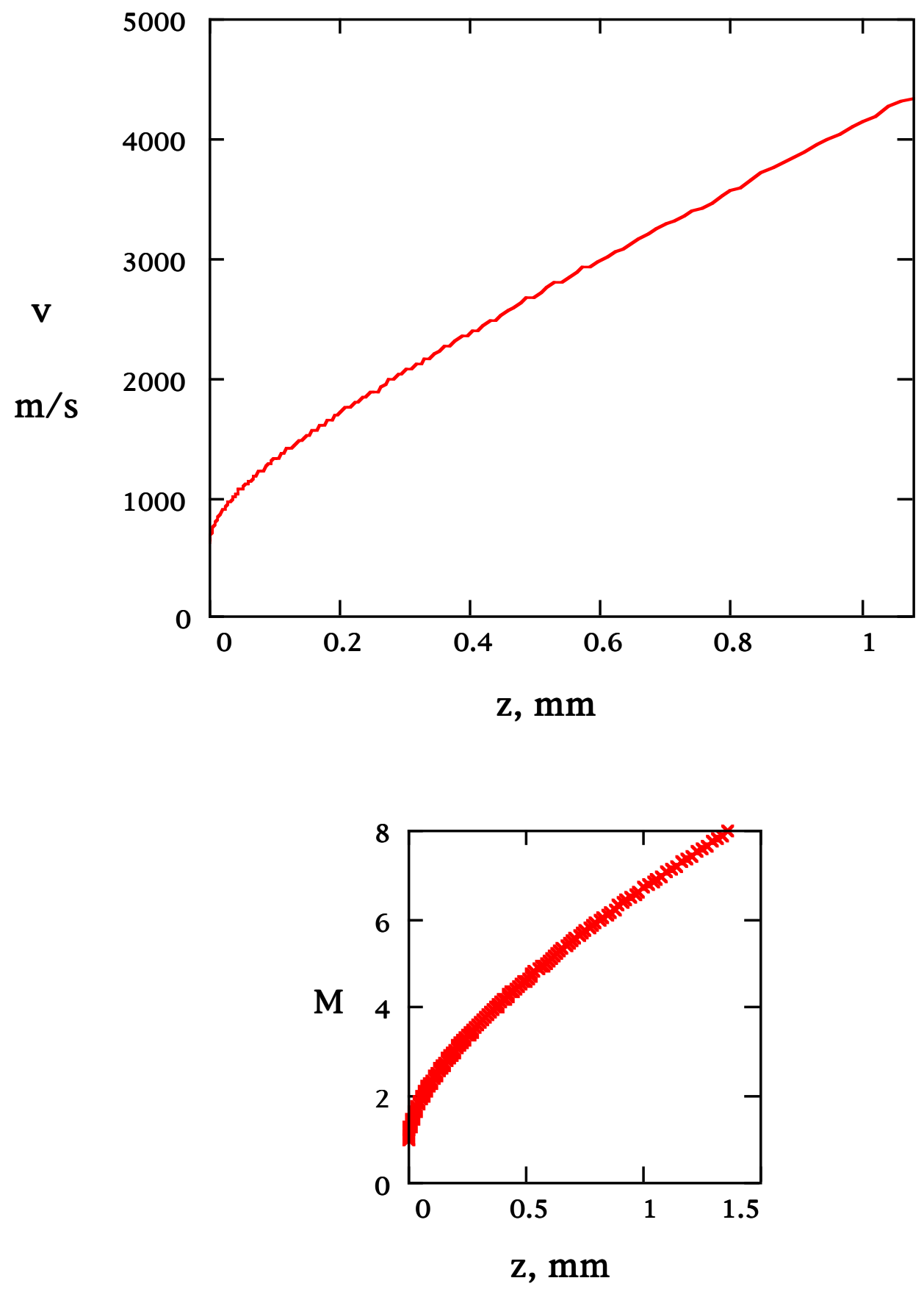

Figure 6, Velocity profile at $275 \mathrm{~ns}$, and $M(z)$ 


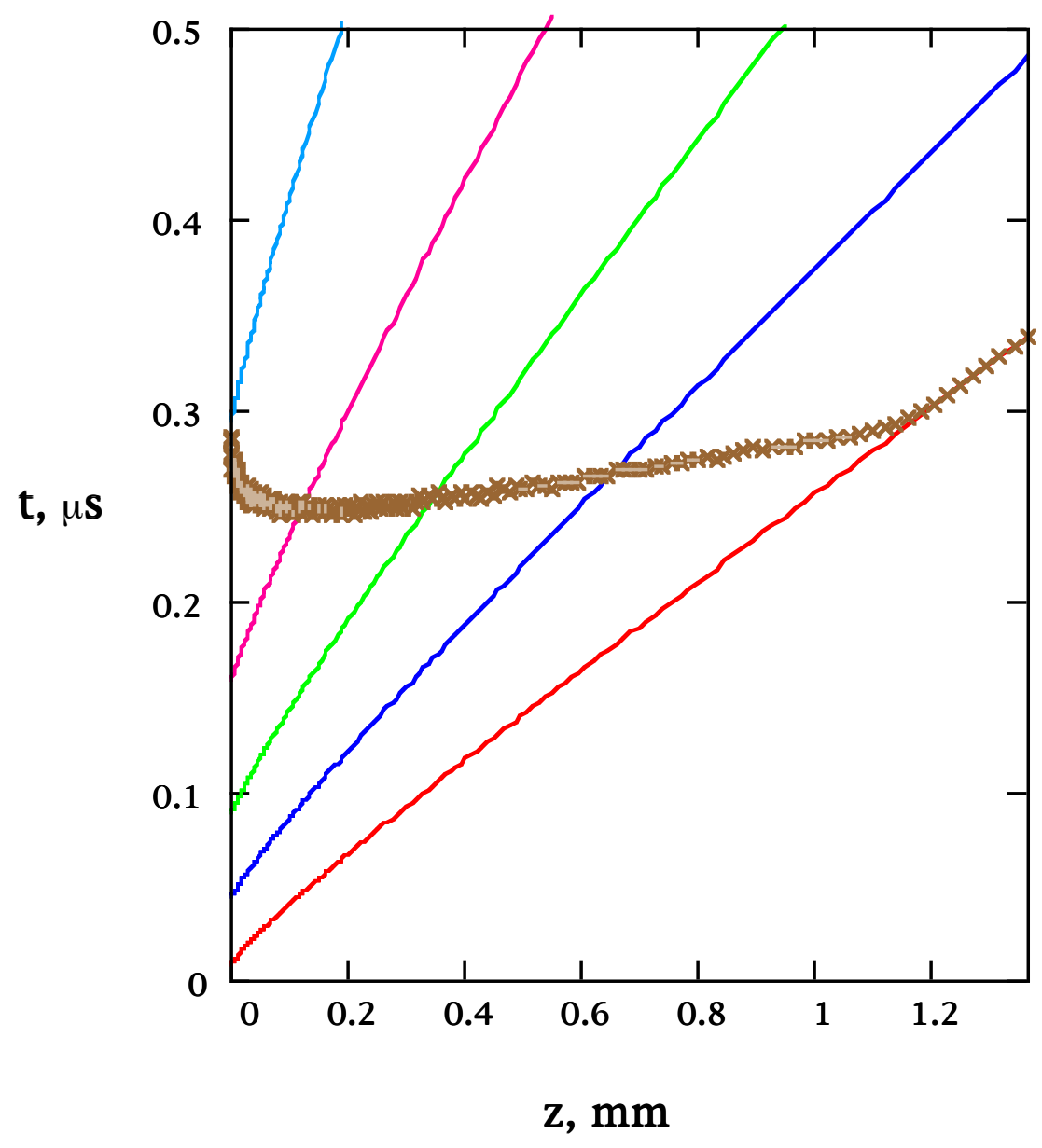

Figure 7, Flow characteristics and the freezing line 


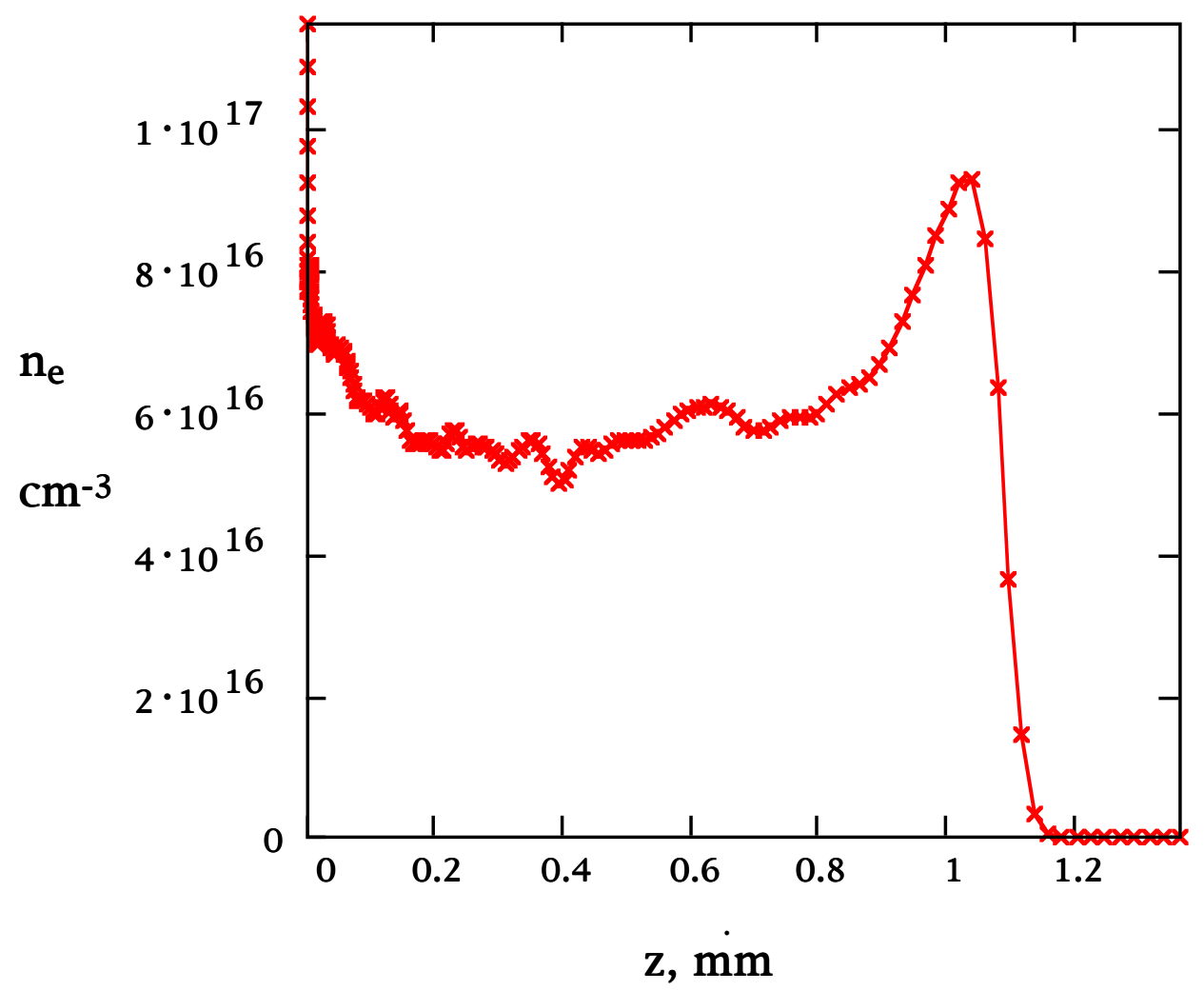

Figure 8, Electron density profile at 275 ns 


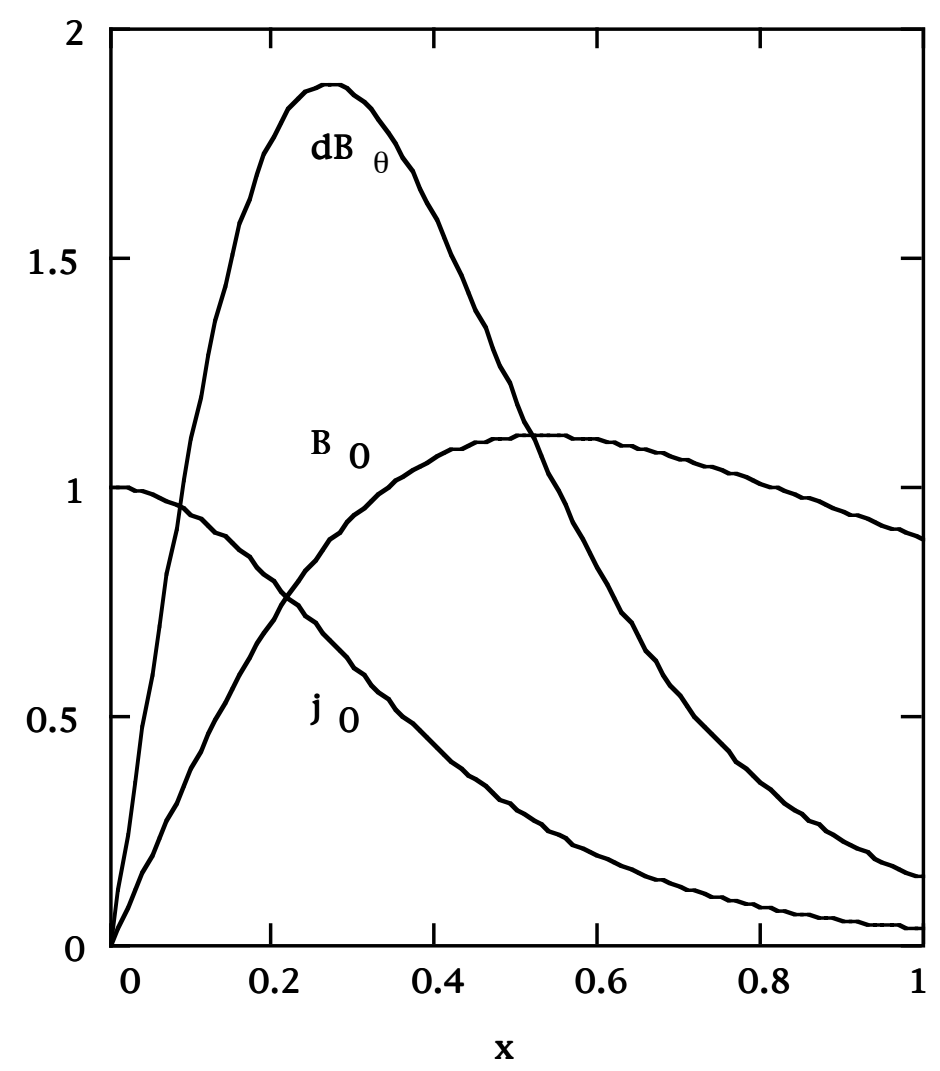

Figure 9, Radial profiles set by electron beam 


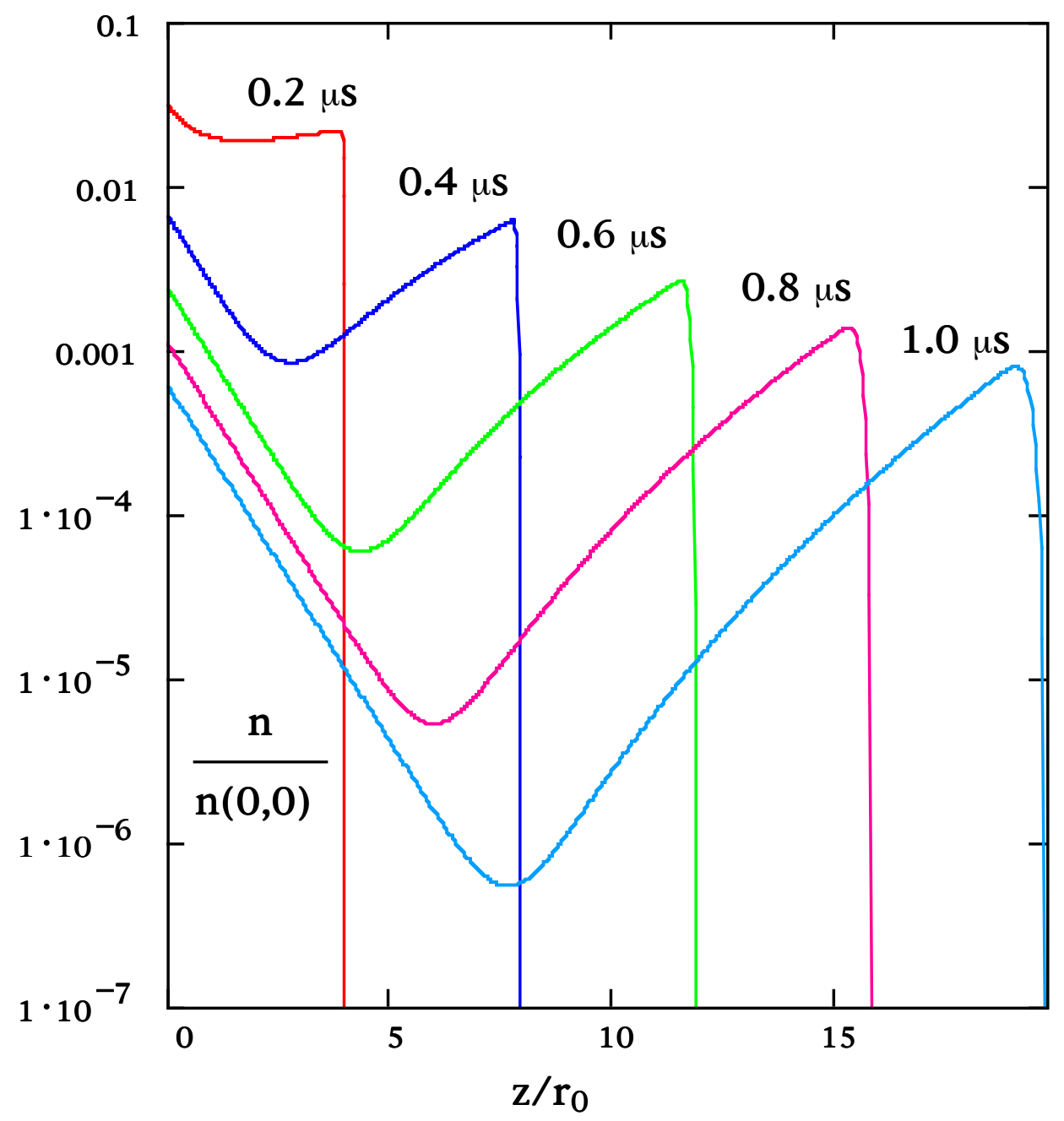

Figure 10, Axial density model, example 1 


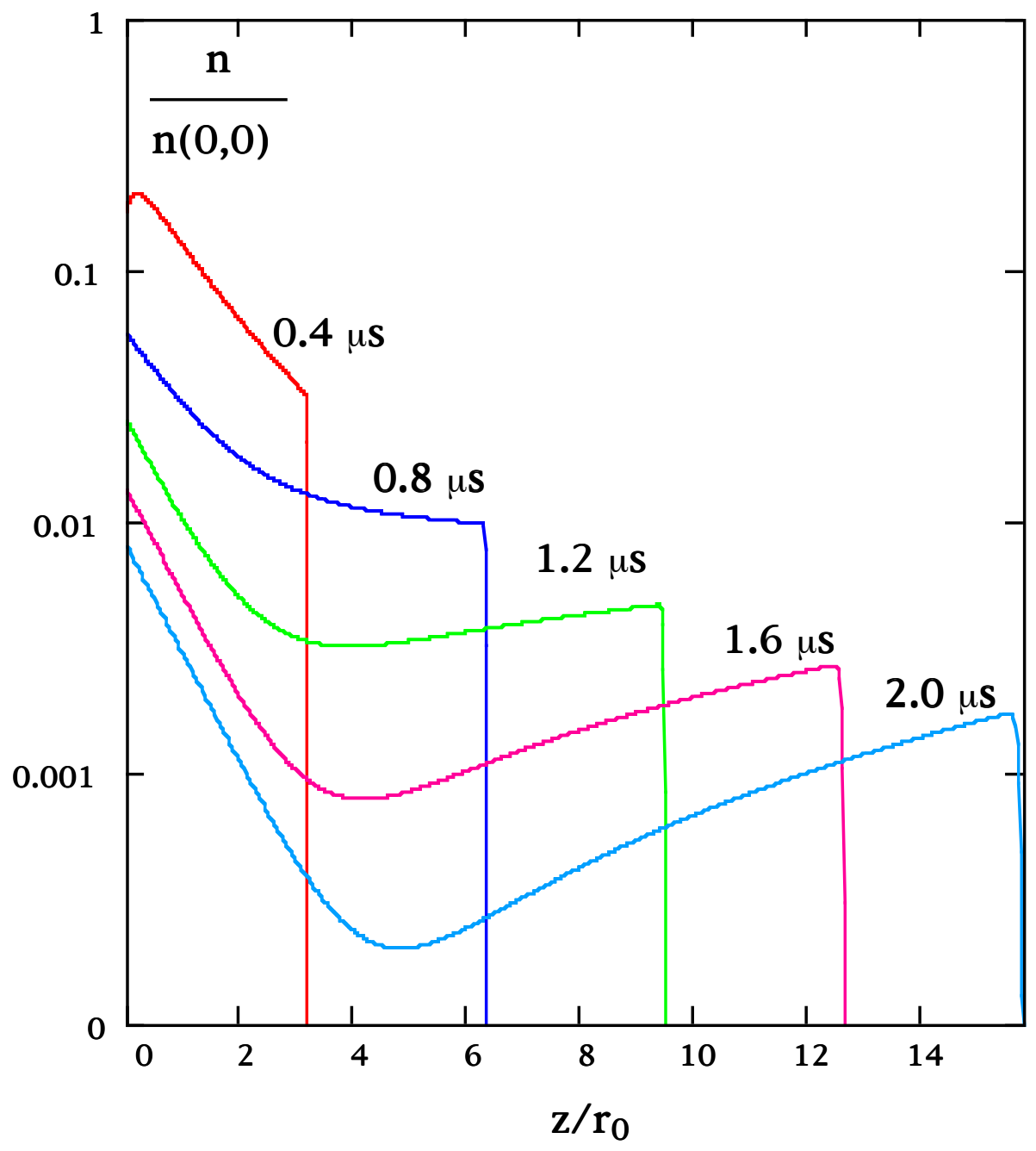

Figure 11, Axial density model, example 2 

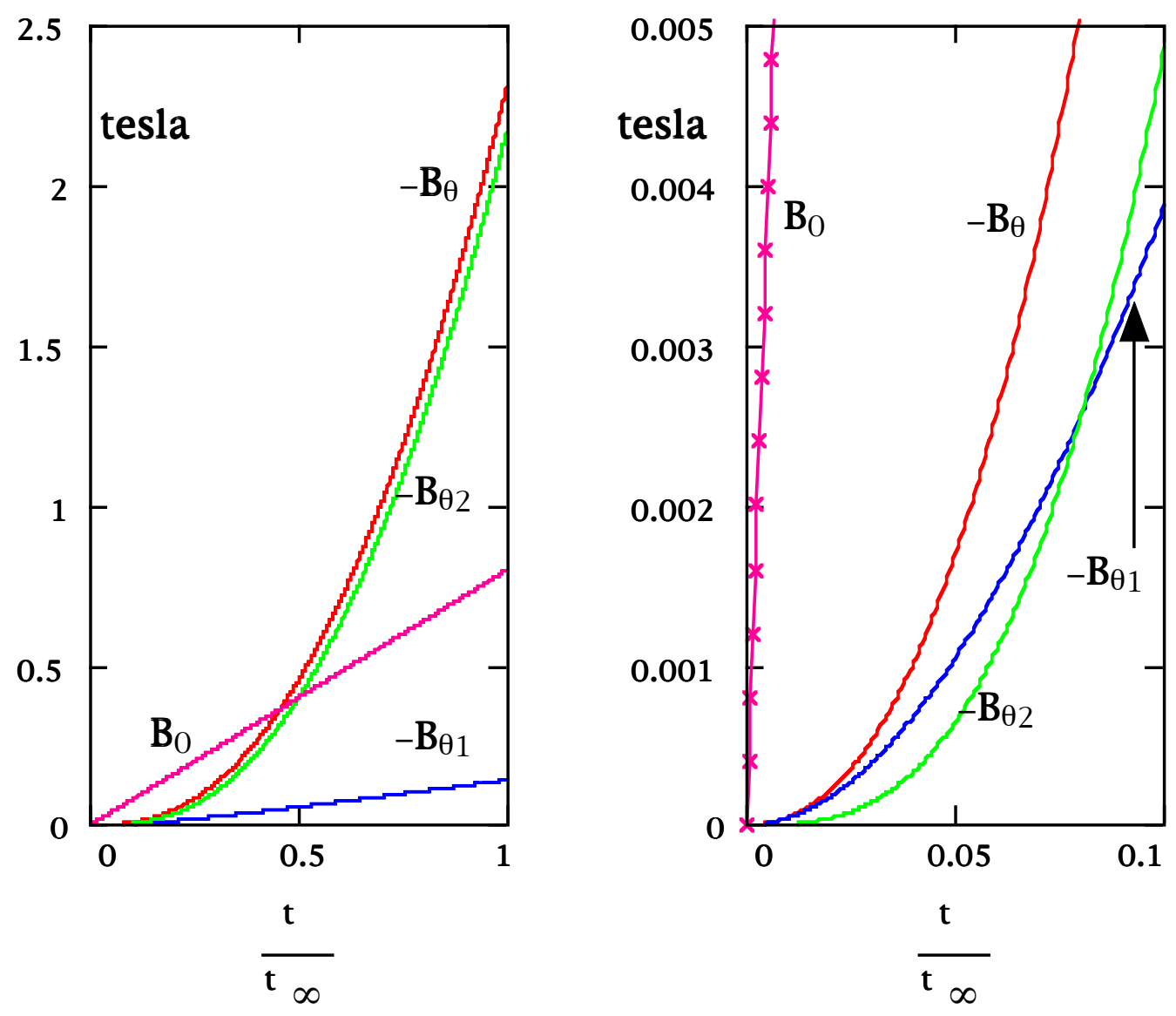

Figure 12, Magnetic field growth during a heating pulse 


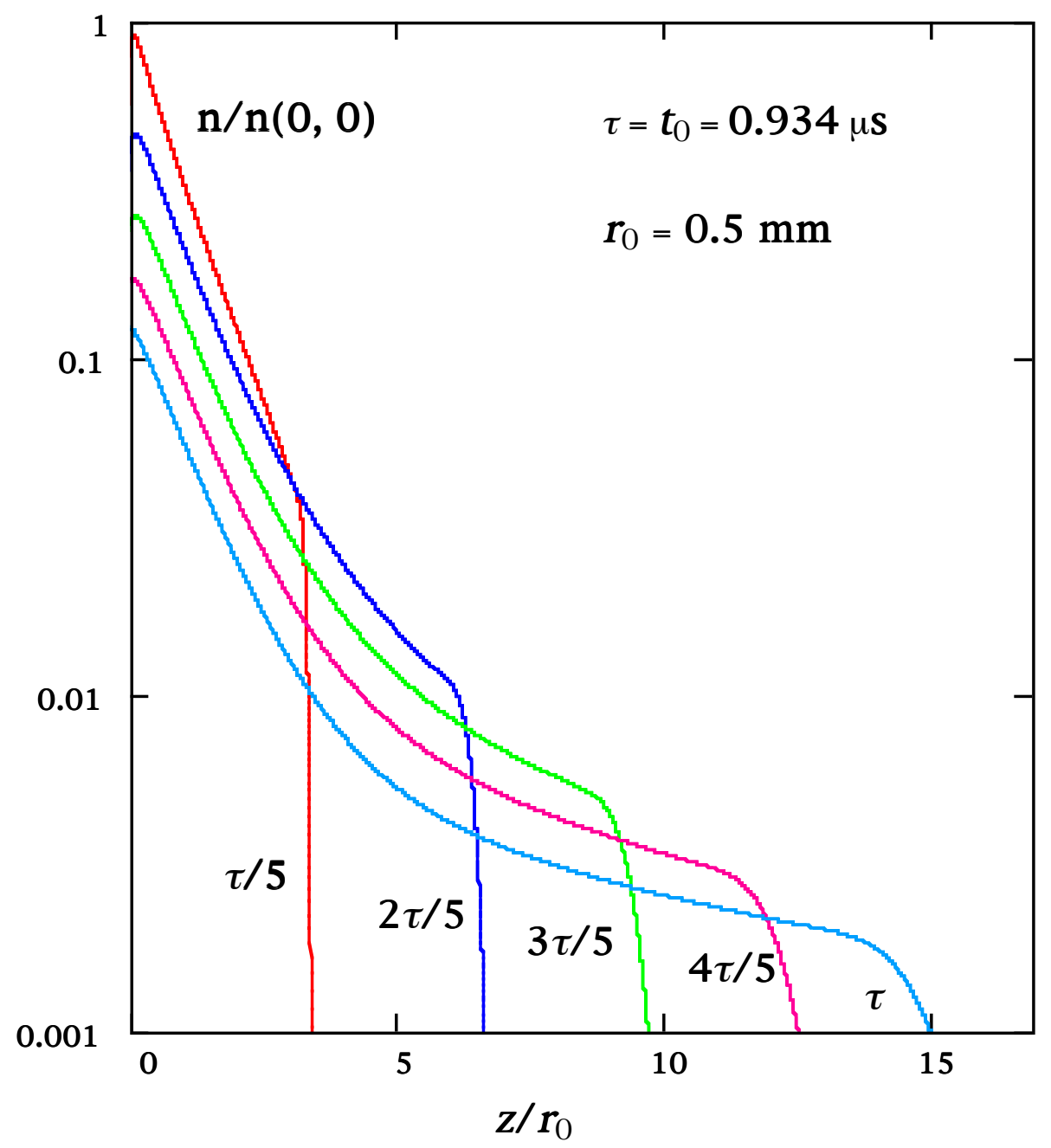

Figure 13, Axial density model, example 3 


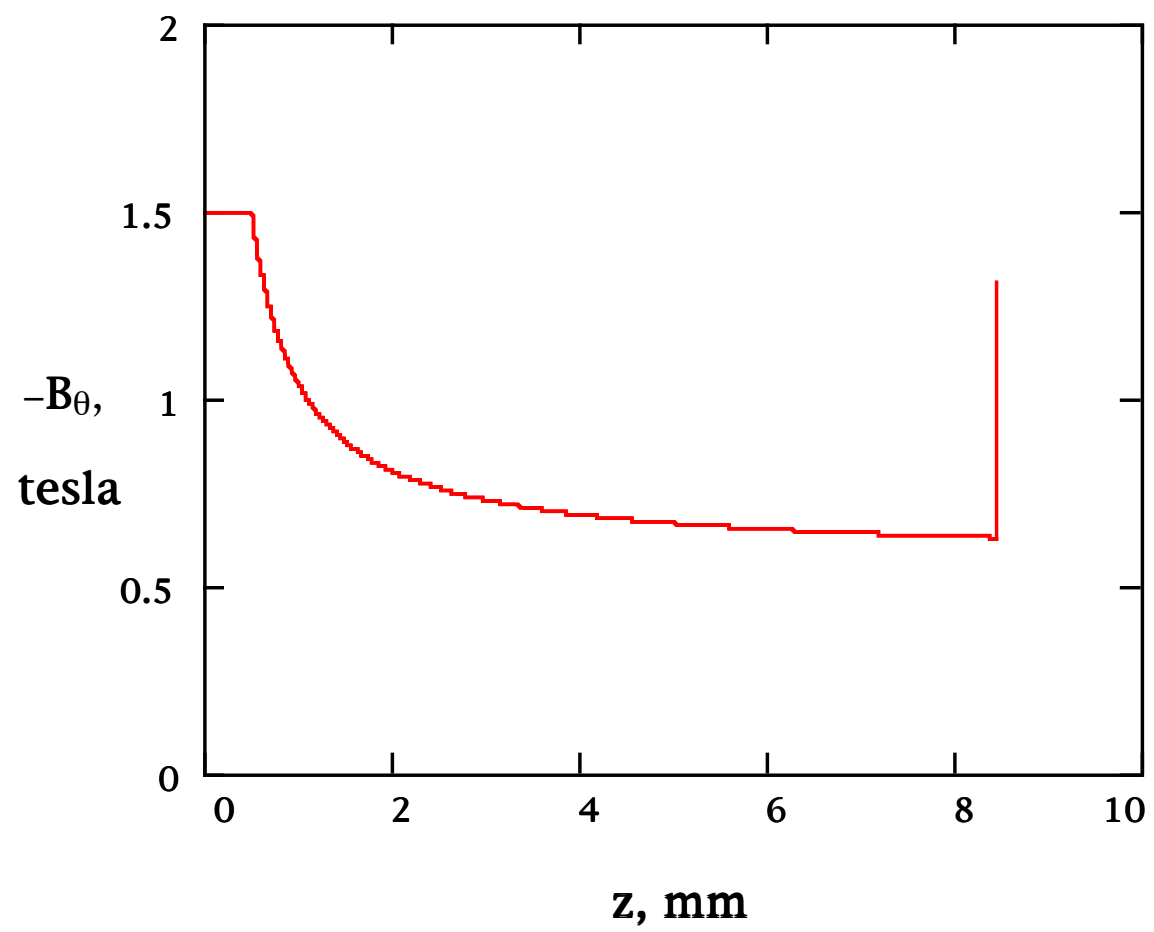

Figure 14, Magnetic induction at 20 ns in example 3 


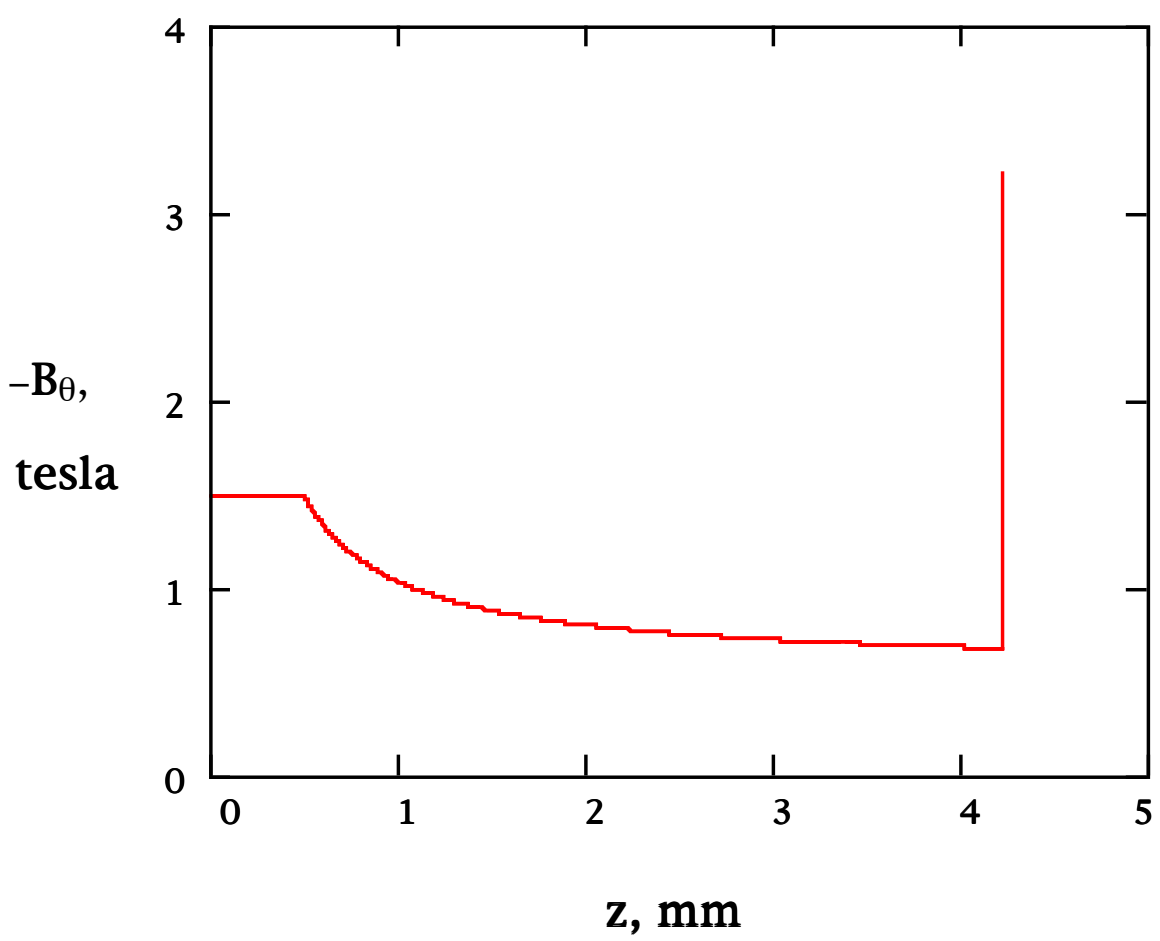

Figure 15, Magnetic induction at 20 ns in example 4 